\title{
Ruxolitinib induces apoptosis of human colorectal cancer cells by downregulating the JAK1/2-STAT1-Mcl-1 axis
}

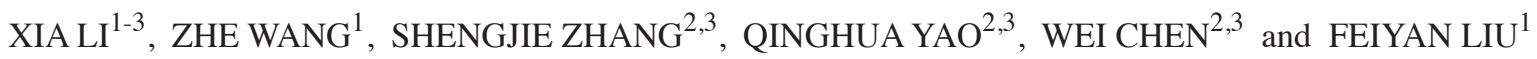 \\ ${ }^{1}$ College of Life Sciences, Zhejiang University, Hangzhou, Zhejiang 310058; ${ }^{2}$ Institute of Cancer and Basic \\ Medicine, Chinese Academy of Sciences; ${ }^{3}$ Zhejiang Cancer Research Institute, Cancer Hospital of The University \\ of Chinese Academy of Sciences (Zhejiang Cancer Hospital), Hangzhou, Zhejiang 310022, P.R. China
}

Received April 8, 2020; Accepted December 14, 2020

DOI: $10.3892 / \mathrm{ol} .2021 .12613$

\begin{abstract}
Under pathological conditions, the Janus kinase (JAK)/STAT signaling pathway can regulate the proliferation, differentiation and migration of tumor cells, including colorectal cancer (CRC). CRC is the third major types of cancer among males and the second among females worldwide. In China, $\mathrm{CRC}$ is the fifth common cancer among both males and females. Western blotting, flow cytometry, RNA interference, immunoprecipitation, xenografts models, and immunohistochemical staining were carried out to evaluate the possible mechanisms of acton of ruxolitinib. The present data suggested that ruxolitinib can suppress CRC cell proliferation by inducing apoptosis. Firstly, JAK1/2-STAT1 was identified as the target of ruxolitinib. Then, ruxolitinib downregulated myeloid cell leukemia-1 (Mcl-1) mRNA level and decreased its protein level, which enabled Bak to trigger CRC apoptosis. Furthermore, ruxolitinib exerted potent activity against CRC xenograft growth in vivo. High expression of phosphorylated STAT1 (S727) was also confirmed in 44 pairs of human colon carcinoma and adjacent normal tissues. Taken together, the results showed that ruxolitinib decreased JAK1/2-STAT1-Mcl-1 protein level and effectively suppressed CRC cell proliferation in vitro and in vivo. Therefore, ruxolitinib could be a promising anticancer agent for CRC treatment.
\end{abstract}

Correspondence to: Dr Wei Chen, Zhejiang Cancer Research Institute, Cancer Hospital of The University of Chinese Academy of Sciences (Zhejiang Cancer Hospital), 1 East Banshan Road, Hangzhou, Zhejiang 310022, P.R. China

E-mail: chenwei@zjcc.org.cn

Professor Feiyan Liu, College of Life Sciences, Zhejiang University, 866 Yuhangtang Road, Hangzhou, Zhejiang 310058, P.R. China E-mail: liuf64@zju.edu.cn

Key words: ruxolitinib, apoptosis, Janus kinase 1/2, phosphorylated STAT1 (S727), myeloid cell leukemia-1, Bak

\section{Introduction}

Colorectal cancer (CRC) is the third most common cancer and the fourth most common cause of cancer-associated death worldwide (1). In China, the incidence of CRC is increasing due to physical inactivity, unhealthy diet, obesity and long-term smoking (2). Over the last decade, colonoscopy screening has significantly improved the 5-year survival rates of patients with CRC. For example, a previous study reported that patients who did not take part in the screening program showed significantly lower cancer-specific survival compared with those who did in the Pudong New Area of Shanghai of China (hazard ratio $(\mathrm{HR})=1.46 ; 95 \%$ confidence interval $(\mathrm{CI})$ : 1.12-1.91) (3-5). Nevertheless, $40 \%$ of patients who undergo surgery, which is applied in combination with chemotherapy or radiation therapy, subsequently experience local and systemic recurrence or resistance (6). Among primary risk factors, complex genomic alterations, which are essential in the mechanism of CRC pathophysiology, may induce resistance of CRC to drug therapy, thus making CRC the fourth most common cause of cancer-associated mortality $(4,7)$. Consequently, investigating the mechanisms underpinning CRC chemosensitivity is important to improve treatment.

Under pathological conditions, the Janus kinase (JAK)-STAT signaling pathway has been shown to regulate proliferation, differentiation and angiogenesis of malignant tumor cells and to promote the development of several malignant tumors, such as breast cancer and esophageal cancer $(8,9)$. This pathway is constitutively activated in myeloproliferative diseases and in various solid tumors, including hepatocellular carcinomas, prostate, breast, head and neck, lung and CRC (10). Although the exact dysregulation mechanism of JAK/STAT signaling in CRC remains unclear, the JAK/STAT signaling pathway has been suggested as therapeutic target for the treatment of CRC $(11,12)$.

Myeloid cell leukemia-1 (Mcl-1) has sequence and functional similarity to Bcl-2 (13). Mcl-1 has a short half-life and is a highly regulated protein (14). The upstream regulatory kinase cascades of Mcl-1 transcription include JAK/STAT, PI3K and mitogen-activated protein kinases (15). Previous studies have suggested that Mcl-1 may have a significant part in the survival or resistance of a variety of cancer cells, such as liver cancer and multiple myeloma $(14,16)$. 
Ruxolitinib (aslo named INCB018424, Jakari) is a small molecule inhibitor of the JAK1/2 kinase, which has been approved by the USA Food and Drug Administration for the treatment of myelofibrosis and polycythemia vera $(17,18)$. Recently, new clinical trials have tested the efficacy of ruxolitinib in the treatment of inflammatory-driven solid tumors (19-21). It was reported that the therapeutic effects observed in patients were not related to JAK mutational capacity and that ruxolitinib initiates only restricted anticlonal activity, thus poignantly altering the inflammatory microenvironment (22). Therefore, ruxolitinib may have potential for the treatment of inflammatory-driven cancer types, such as pancreatic cancer and CRC. The present study aimed to investigate the inhibitory mechanisms of ruxolitinib on CRC cells both in vitro and in vivo.

\section{Materials and methods}

Reagents. Primary antibodies against caspase-3 (cat.no.9662S), cleaved caspase-3 (cat. no. 9664), caspase-9 (cat. no. 9502), cleaved caspase-9 (cat. no. 9501), caspase-8 (cat. no. 4790), PARP (cat. no. 9532), cleaved PARP (cat. no. 9541), Mcl-1 (cat. no. 5453), Bcl-2 (cat. no. 2872), Bcl-xL (cat. no. 2764), Bak (cat. no. 12105), JAK1 (cat. no. 3344), JAK2 (cat. no. 3230), STAT1 (cat. no. 9172) and $\beta$-actin (cat. no. 4970) were acquired form Cell Signaling Technology, Inc. Anti-Mcl-1 antibody (cat. no. S-19), control siRNA-A (cat. no. sc-37007), and STAT1p84/p91 siRNA (h) (cat. no. sc-44123) were from Santa Cruz Biotechnology, Inc. Anti-STAT1 [phosphorylated (p)S727] (cat. no. ab109461) antibody was acquired from Abcam. Annexin V-FITC was purchased from BD Bioscience. Z-VAD-FMK was from Selleck Chemicals. Monoclonal antibody anti-Bak (cat. no. TC-100) was from Merck KGaA. JC-1 dye was purchased from AAT Bioquest, Inc.

Cell line and culture conditions. Human CRC cell lines LS411N, SW620, DLD-1, HCT116, SW480 and mouse CRC cell line, CT-26, were obtained from the American Type Culture Collection. Cells were cultured in Dulbecco's Modified Eagle's Medium supplemented with 10\% FBS (both purchased from Gibco, Thermo Fisher Scientific), $100 \mathrm{U} / \mathrm{ml}$ of penicillin and $100 \mu \mathrm{g} / \mathrm{ml}$ of streptomycin (cat. no. 15070-063, Gibco; Thermo Fisher Scientific, Inc.) in a humidified atmosphere containing $5 \% \mathrm{CO}_{2} / 95 \%$ air at $37^{\circ} \mathrm{C}$.

Cell viability assay. An MTT assay was used for determining ruxolitinib cytotoxicity against CRC cells. Briefly, 2,000 cells/well were plated in 96-well plates and incubated for $24 \mathrm{~h}$ and then exposed to gradually increasing concentrations $(0,2.5,5,10,15,20,25,30,40,50 \mu \mathrm{M})$ of ruxolitinib for $48 \mathrm{~h}$. MTT solution (prepared in serum-free growth medium) was added to each well and was incubated for another $4 \mathrm{~h}$ at $37^{\circ} \mathrm{C}$. DMSO was added and the absorbance at $570 \mathrm{~nm}$ was determined using a microplate reader. CCK-8 assay and real-time monitoring of cell viability using an $\mathrm{xCELLigence} \mathrm{system} \mathrm{were} \mathrm{performed} \mathrm{to} \mathrm{determine} \mathrm{the} \mathrm{cyto-}$ toxicity of ruxolitinib against CRC cells. Briefly, LS411N and SW620 cells were seeded into 96-well plates at a density of 2,000 cells/well, incubated for $24 \mathrm{~h}$ at $37^{\circ} \mathrm{C}$ and then exposed to different concentrations $(0,2.5,5,10,15,20,25,30,40$,
$50 \mu \mathrm{M}$ ) of ruxolitinib for $48 \mathrm{~h}$ at $37^{\circ} \mathrm{C}$. CCK-8 was added to the plates and incubated for $1 \mathrm{~h}$ at $37^{\circ} \mathrm{C}$. The absorbance was measured at a wavelength of $570 \mathrm{~nm}$, using a microplate reader. The in vitro cell growth assay was performed on an xCELLigence system from ACEA Biosciences Inc., using E-plates. Briefly, the E-plates with $50 \mu \mathrm{l}$ of growth medium in each well were placed into the RTCA-DP instrument for baseline test. The LS411N and SW620 cells were seeded into the plates at a density of 5,000 cells/well in $100 \mu \mathrm{l}$ of growth medium and treated with indicated concentrations of ruxolitinib. The E-plates were incubated at room temperature for $30 \mathrm{~min}$ and placed into the RTCA-DP instrument for data collection at $15 \mathrm{~min}$ intervals.

Early/late apoptosis analyzed by flow cytometry. After treatment with various concentrations of ruxolitinib for 48, 72, $96 \mathrm{~h}$, human CRC LS411N, SW620 cells were collected and washed with ice-cold PBS before staining with Annexin V-FITC for $15 \mathrm{~min}$ at room temperature in the dark followed by PI staining for $5 \mathrm{~min}$ at room temperature. The samples were analyzed with a BD FACSCanto flow cytometer (BD Biosciences). Data analysis was performed by the software WinMDI 2.9 (BD Biosciences). Human CRC LS411N and SW620 cells were treated with Z-VAD-FMK for $1 \mathrm{~h}$ followed by ruxolitinib treatment for another $48 \mathrm{~h}$, and were collected and analyzed as aforementioned.

Western blotting. LS411N, SW620 cells were lysed in Laemmli lysis buffer (Merck, cat. no. S3401). Proteins (25 $\mu \mathrm{g} /$ lane) were loaded in denaturing $12 \%$ or $15 \%$ SDS-PAGE gels and transferred to nitrocellulose membranes. The membranes were then incubated with 5\% non-fat milk for $2 \mathrm{~h}$ at room temperature, washed with PBS containing $0.1 \%$ Tween-20 and incubated with primary antibodies (1:1,000 dilution) overnight at $4^{\circ} \mathrm{C}$. After washing with PBST the membrane was incubated with secondary antibodies conjugated with horseradish peroxidase (1:2,000 dilution) for $2 \mathrm{~h}$ at room temperature. Signals were visualized using the Pierce ECL Plus kit (cat. no. 32132) with a chemiluminescence system (Amersham; Cyvita). $\beta$-actin was used as a loading control.

Mitochondrial membrane potential assay. To examine the mitochondrial membrane potential, JC-1 staining was used. Human CRC LS411N, SW620 cells were incubated with various concentrations of ruxolitinib for $48 \mathrm{~h}$ as aforementioned. Then, the cells were harvested, washed with PBS and resuspended in JC-1 staining solution at room temperature for 10 min. Cells were detected by a BD FACSCanto flow cytometer (BD Biosciences). Data analysis was performed using WinMDI 2.9 software (BD Biosciences).

Plasmid construction and transfection. The coding sequences of human Mcl-1 mRNA were amplified by PCR using total cDNA as previously described (14). The PCR products were digested with HindIII and EcoRI and subcloned into the pcDNA3.1 vector (obtained from co-author Shengjie Zhang's lab). The integrity of the respective plasmid constructs was confirmed using PCR. One microgram pcDNA3.1-negative control $(0.2 \mu \mathrm{g} / \mathrm{ml})$ and pcDNA3.1/Mcl-1 $(0.2 \mu \mathrm{g} / \mathrm{ml})$ was transfected in SW620 and LS411N cells using Lipofectamine ${ }^{\circledR} 2000$ 
(Invitrogen; Thermo Fisher Scientific, Inc.) at $37^{\circ} \mathrm{C}$ for $24 \mathrm{~h}$. After $24 \mathrm{~h}$, the LS411N and SW620 cells were used for subsequent experiments.

Reverse transcription-quantitative (RT-q)PCR analysis. Total RNA was isolated from LS411N and SW620 cells using TRIzol ${ }^{\circledR}$ reagent (Invitrogen; Thermo Fisher Scientific, Inc.), according to the manufacturer's protocols (23). Complementary DNA synthesis was performed using the PrimeScript RT Reagent kit (Takara Bio, Inc.). Real-time PCR amplification was performed using the ABI 7300 Fast Real-Time PCR system (Applied Bioscience; Thermo Fisher Scientific, Inc.), according to the manufacturer's instructions. The amplification reactions were performed using $1 \mathrm{X}$ Power SYBR Green PCR Master mix (Applied Biosystems; Thermo Fisher Scientific, Inc.). PCR primers for Mcl-1 (forward, 5'-GCTTCG GAAACTGGACAT-3'; reverse, 5'-CACAAACCCATCCCA GCC-3'), $\beta$-actin (forward, 5'-ACACCCCAGCCATGTACG TT-3'; reverse, 5'-TCACCGGAGTCCATCACGAT-3') were designed using Primer Premier 5 software (Premier Biosoft International). The standard temperature profile, including initial denaturing, annealing and extension, was used as previously described (14).

RNA interference of STATI. Short interfering (si)RNAs for STAT1 (cat. no. sc-44123) and non-targeting siRNA negative control (cat. no. sc-37007) were obtained from Santa Cruz Biotechnology, Inc. LS411N and SW620 cells were transfected with $1 \mu \mathrm{g}$ siRNA $(0.2 \mu \mathrm{g} / \mathrm{ml})$ using Lipofectamine 2000 reagent at $37^{\circ} \mathrm{C}$ for $24 \mathrm{~h}$, according to the manufacturer's instructions. Before treatment, cells were incubated for $24 \mathrm{~h}$ and the silencing efficiency of the siRNA was determined using western blotting as aforementioned. Subsequent experiments were performed $24 \mathrm{~h}$ post-transfection.

Measurement of Bak conformational change. LS411N and SW620 cells were fixed in $4 \%$ paraformaldehyde for $10 \mathrm{~min}$ at room temperature, washed three times with PBS and permeabilized with $0.1 \%$ PBS-Tween-20 for $20 \mathrm{~min}$. Cells were then incubated with anti-active Bak for $30 \mathrm{~min}$ at $4{ }^{\circ} \mathrm{C}$, followed by incubation with 1:200 dilution of FITC-conjugated goat-anti-mouse IgG (Santa Cruz Biotechnology, Inc. cat. no. sc-2010) for $30 \mathrm{~min}$ at $4^{\circ} \mathrm{C}$ and analyzed using a BD FACSCanto flow cytometer (BD Biosciences). Data analysis was performed using WinMDI 2.9 software (BD Biosciences).

Immunoprecipitation. Protein-protein interactions were determined by co-immunoprecipitation (Co-IP) analysis. LS411N and SW620 cells were lysed in co-immunoprecipitation lyse-bind-wash buffer [50 mM Tris-HCl, $150 \mathrm{mM} \mathrm{NaCl}, 1 \%$ NP-40 (V/V), 2 mM EDTA] containing 1\% protease inhibitor cocktail (Pierce; Thermo Fisher Scientific, Inc.) at $4^{\circ} \mathrm{C}$ with rotation for $60 \mathrm{~min}$. The lysate was then centrifuged at $12,000 \mathrm{x} \mathrm{g}$ for $10 \mathrm{~min}$ at $4^{\circ} \mathrm{C}$. After that, samples $(800 \mu \mathrm{g}$ protein for control or indicated concentrations of ruxolitinib treatment were incubated with anti-Bak antibody at $4^{\circ} \mathrm{C}$ overnight. A total of $50 \mu \mathrm{l}$ Protein $\mathrm{A} / \mathrm{G}$ agarose was added to each sample and incubated for another $4 \mathrm{~h}$ at $4^{\circ} \mathrm{C}$. Samples were washed three times and subjected to immunoblot analysis using anti-Mcl-1 antibody as aforementioned.
Animals and antitumor activity in vivo. In total, $14 \mathrm{Balb} / \mathrm{c}$ nude mice, male, 6 weeks old, weighing 20-25 g, were obtained from Vital River Laboratories. All the animals were housed in an environment with temperature of $22 \pm 1^{\circ} \mathrm{C}$, relative humidity of $50 \pm 1 \%$ and a light/dark cycle of $12 / 12 \mathrm{~h}$ and allowed access ad libitum to water and diet. All animal studies (including the mice euthanasia procedure) were approved and performed in compliance with the regulations and guidelines of Zhejiang University Institutional Animal Care Committee (Hangzhou, China; approval no. ZJU20180108020) and conducted according to the Association for Assessment and Accreditation of Laboratory Animal Care International and the Institutional Animal Care and Use Committee guidelines.

Human CRC LS411N xenografts were established by subcutaneously injecting $1 \times 10^{7}$ cells into the right flank of the nude mice. When the tumors were palpable, the tumor-bearing mice were randomized into two groups: Treatment group and control group with seven mice/group. The treatment group received ruxolitinib (150 mg/kg body weight) by oral gavage every 2 days for 14 days, while the control group received the same amount of saline. At the end of experiment, mice were anesthetized with an intraperitoneal injection of sodium pentobarbital (50 mg/kg body weight) and euthanized by cervical dislocation.

Tumor size was measured using a micrometer caliper. Tumor volume $\left(\mathrm{mm}^{3}\right)$ was calculated using the following formula: Length $(\mathrm{mm}) \mathrm{x}$ width $(\mathrm{mm}) \mathrm{x}$ width $(\mathrm{mm}) / 2$. Tumor volume data are expressed as the mean $\pm \mathrm{SEM}$, while the rest of the data are presented as mean \pm SD. In addition, body weight for each mouse was measured to evaluate the toxicity of the treatment. Moreover, blood was collected at end of time point. The blood samples were centrifuged at $500 \mathrm{xg}$ for $5 \mathrm{~min}$ at $4^{\circ} \mathrm{C}$, and then the serum was collected. Serum creatine kinase and alanine aminotransferase levels were detected using ELISA kits (creatine kinase, Biovision, cat. no. E4607) and alanine aminotransferase (Biovision, cat. no. K752), according to the manufacturer's instructions.

Immunohistochemistry. In total, 44 pairs of human CRC biopsy and adjacent normal tissue specimens (5/20/2009-08/05/2015) were collected from patients at Zhejiang Cancer Hospital (Hangzhou, China). This included 32 men and 12 women, with an age range of 27-76 years and median age of 57.5 years. The normal tissue specimens were collected from the far end of the surgical tissues ( $\geq 5 \mathrm{~cm}$ from tumor tissues) All patients were provided written informed consent for the project, which was approved by the Medical Ethics Committee of Zhejiang Cancer Hospital (Hangzhou, China). The tissue chip was made and the hematoxylin and eosin was staining by Servicebio Company, Hangzhou (https://www.servicebio.cn).

For hematoxylin and eosin staining, the paraffinized sections were dewaxed using dimethylbenzene, $100 \%$ ethanol and $75 \%$ ethanol followed by tap water rinse. Sections were subsequently stained with hematoxylin solution for 3-5 min at room temperature followed by tap water rinse. The samples were treated with Hematoxylin Differentiation solution and Hematoxylin Scott Tap Bluing (cat. no. G1003; Wuhan Servicebio Technology Co., Ltd.), according to the manufacturer's protocol, and then rinsed with tap water, $85 \%$ ethanol and 95\% ethanol. The sections were stained with 
eosin dye for $5 \mathrm{~min}$ at room temperature. The sections were dehydrated with $100 \%$ ethanol (three times) and dimethylbenzene (two times). The sections were cover slipped and sealed with neutral gum.

For immunohistochemistry analysis, tissues were deparaffinized, rehydrated and incubated in 3\% hydrogen peroxide in $\mathrm{H}_{2} \mathrm{O}$. Heat-induced antigen retrieval was carried out for all sections in $0.01 \mathrm{M}$ citrate buffer, $\mathrm{pH}$ 6.0, using a steamer at $95^{\circ} \mathrm{C}$. All aforementioned primary antibodies were diluted with PBST to a concentration of 1:50 and were applied to the sections. Incubation lasted for $30 \mathrm{~min}$ at room temperature followed by incubation with a DakoEnVision + System-HRP Labelled Polymer (Dako; Agilent Technologies) for $10 \mathrm{~min}$ at room temperature. Diaminobenzidine was then applied for $10 \mathrm{~min}$ at room temperature. The sections were counterstained for $5 \mathrm{~min}$ with hematoxylin, dehydrated (including $3 \mathrm{~min}$ with $75 \%$ ethanol, 3 min with $95 \%$ ethanol, 3 min with absolute ethanol, 3 min with dimethylbenzene, and another 3 min with dimethylbenzene at room temperature), cover slipped and visualized at room temperature. Slides were blindly scored by two independent gastrointestinal pathologists. Samples were evaluated for staining using the following scale: Scarce positive cells $=0$, low abundance of positive cells $=1$, moderate abundance of positive cells $=2$ and high abundance of positive cells $=3+$. The two sets of scores were combined for the highest possible score of 6 .

Statistical analysis. Data are expressed as the mean \pm SD or SEM from at least three independent experiments. Immunohistochemistry data were statistically analyzed using the Wilcoxon matched-pairs test. All statistical analyses were performed using GraphPad Prism 5.0 (GraphPad Software, Inc.). Statistical significance was determined using an ANOVA followed by Tukey's post hoc test, and $\mathrm{P}<0.05$ was considered to indicate a statistically significant difference.

\section{Results}

Ruxolitinib induces CRC cell apoptosis. The cytotoxicity of ruxolitinib on LS411N, CT-26, SW620, DLD-1, HCT116 and SW480 cells was examined using MTT assays. As shown in Fig. 1A and Table I, all cancer cell lines were sensitive to ruxolitinib with the $\mathrm{IC}_{50}$ ranging from 8 to $25 \mu \mathrm{M}$. To confirm the cytotoxicity of ruxolitinib on LS411N and SW620 cells, CCK-8 assays and real-time monitoring of cell viability using an xCELLigence system were performed (24). As presented in Fig. S1, ruxolitinib significantly inhibited LS411N and SW620 cell proliferation in a dose-dependent manner.

To elucidate the mechanism underlying ruxolitinib-mediated suppression of CRC cell proliferation, two sensitive cell lines were selected, LS411N and SW620. Ruxolitinib induced discernible apoptosis in both cell lines in a dose-dependent manner, with an increased percentage of Annexin V-positive cells (Fig. 1C and D). The results of long-term apoptotic assay are shown in Fig. S2. A time-dependent manner was observed between 72 and $96 \mathrm{~h}$ treatments in both two cell lines. In addition, increased expression of cleaved caspase 3, 8, 9 and PARP (Fig. 1B) was observed in the LS411N and SW620 cells $48 \mathrm{~h}$ after incubation with increasing concentration of ruxolitinib. To further confirm ruxolitinib-induced apoptosis,
Table I. Cytotoxicity of ruxolitinib against human colorectal cancer cells.

\begin{tabular}{lr}
\hline Cell line & $\mathrm{IC}_{50}(\mu \mathrm{M})$ \\
\hline LS411N & $8.44 \pm 0.42$ \\
SW620 & $13.42 \pm 0.47$ \\
SW480 & $19.70 \pm 1.71$ \\
HCT116 & $18.41 \pm 0.20$ \\
DLD1 & $20.90 \pm 1.51$ \\
CT26 & $21.50 \pm 0.80$
\end{tabular}

Data are presented as the mean \pm standard deviation of three independent experiments.

LS411N and SW620 cells were cultured with pan-caspase inhibitor Z-VAD-FMK. As expected, Z-VAD-FMK blocked ruxolitinib-induced apoptosis (Fig. 1E-H).

Furthermore, another biochemical marker of apoptosis pathways, cytochrome c was investigated. The western blot analysis indicated that ruxolitinib treatment induced the release of cytochrome $\mathrm{c}$ in a dose-dependent manner (Fig. 1I). JC-1 staining together with flow cytometry analysis was used to determine the effect of ruxolitinib on mitochondrial outer membrane permeabilization (MOMP). As shown in Fig. 1J and K, the disruption of MOMP induced by ruxolitinib was upregulated compared with the control. After treatment with 20 and $30 \mu \mathrm{M}$ ruxolitinib, the percentage of cells with depolarized MOMP increased to $9.50 \pm 1.84$, and $11.85 \pm 0.21 \%$ in LS411N and SW620 cells, respectively. These data suggested that ruxolitinib inhibits human CRC cell proliferation through an apoptosis-dependent mechanism.

Mcl-1 is the molecular target of ruxolitinib. It was demonstrated that ruxolitinib partly suppressed CRC cell proliferation by inducing apoptosis through activating the intrinsic pathway. Bcl-2 family proteins that are key mediators of the intrinsic apoptosis pathway were screened out $(25,26)$. As shown in Fig. 2A, ruxolitinib selectively decreased the Mcl-1 protein level in LS411N and SW620 cells in dose- and long time-dependent manner, rather than short time-dependent within $6 \mathrm{~h}$, suggesting that Mcl-1 was a molecular target of ruxolitinib.

To further explore whether the ruxolitinib-mediated decrease of Mcl-1 occurred at the transcriptional level, RT-PCR was used to identify the effects of ruxolitinib on Mcl-1 mRNA. The results showed that the Mcl-1 mRNA level was markedly reduced in LS411N and SW620 cells compared with the control (Fig. 2B). To determine whether decreased Mcl-1 mRNA was due to reduced Mcl-1 mRNA stability, ruxolitinib was incubated with or without actinomycin $\mathrm{D}$, a transcription inhibitor (27), in LS411N and SW620 cells. As shown in Fig. S3, there was no significant difference between Mcl-1 mRNA level in the actinomycin D alone and combination group. These results suggested that ruxolitinib-mediated Mcl-1 decrease was more likely to occur at the transcriptional level without affecting stability of Mcl-1 mRNA. 


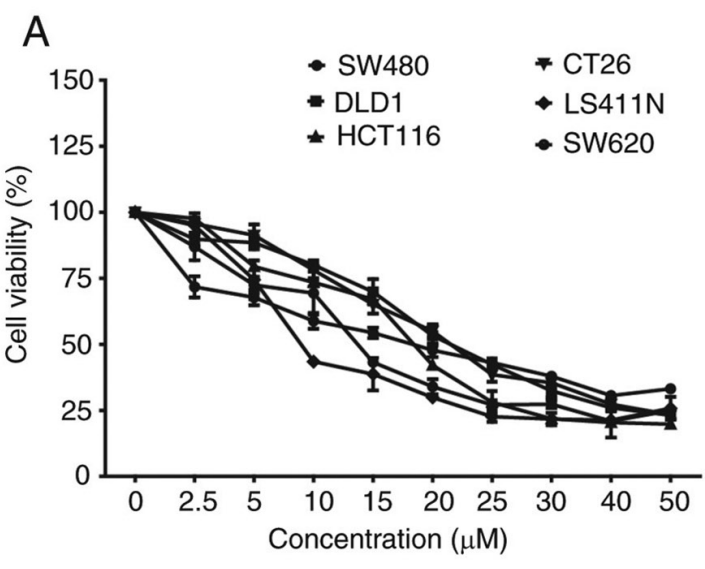

C

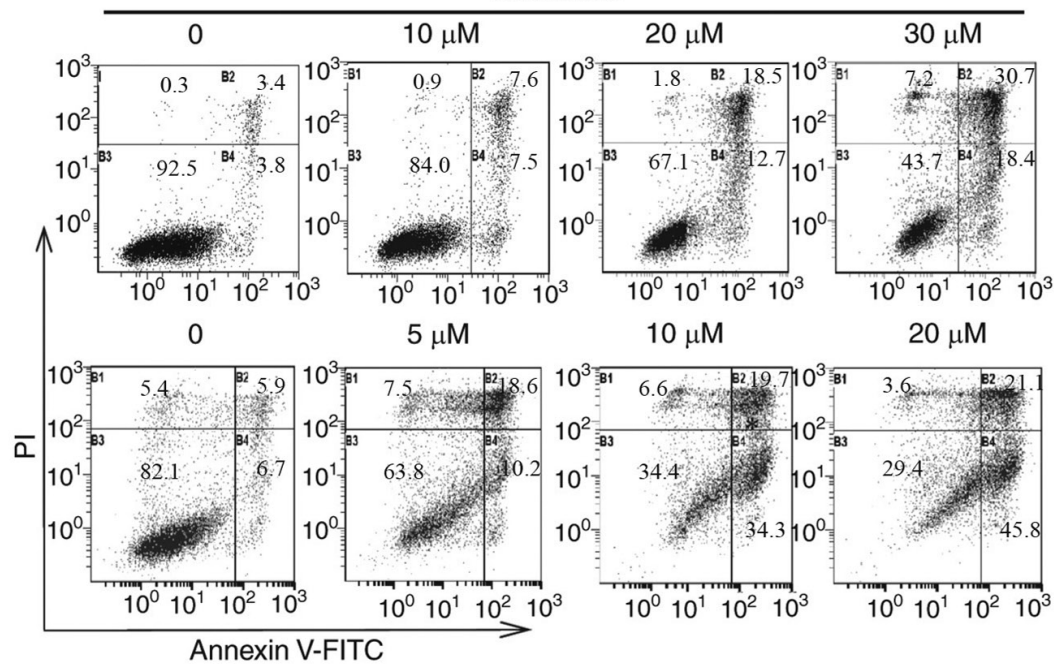

B

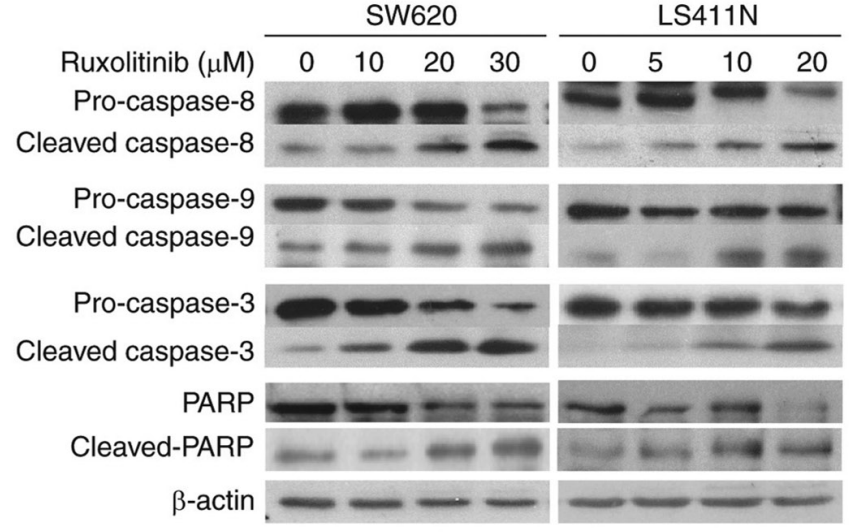

D
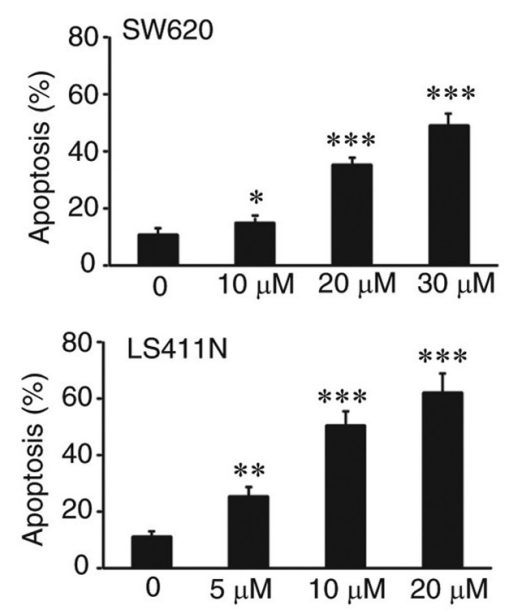

E

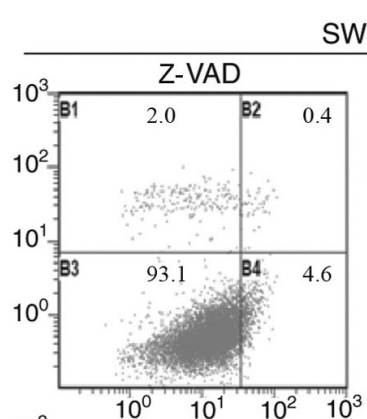

SW620
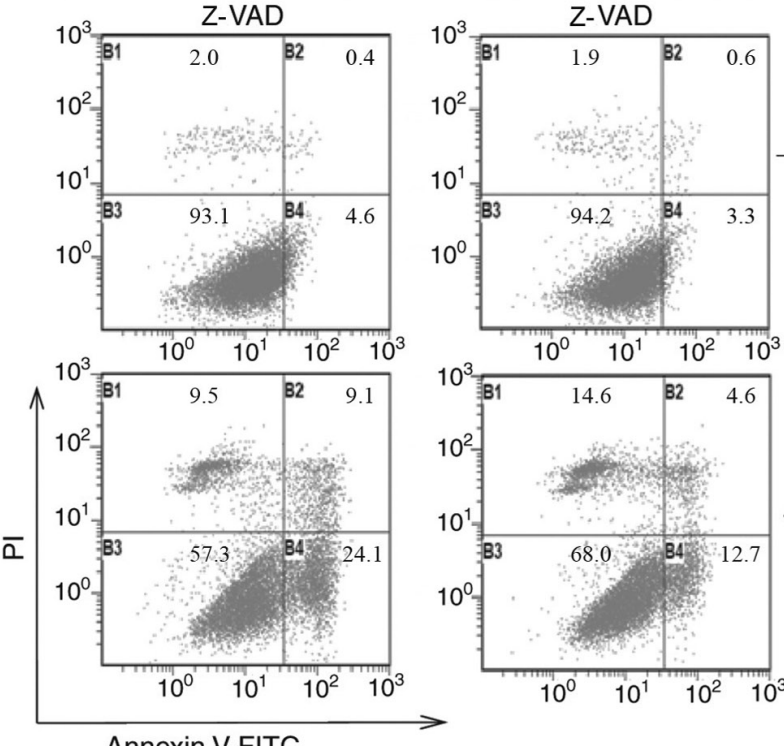

-Ruxolitinib

F

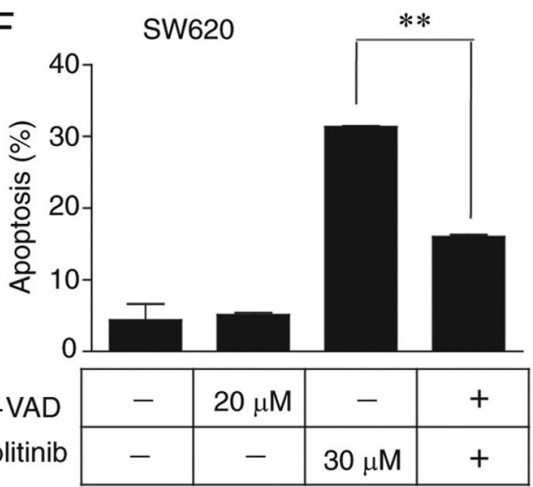

Annexin V-FITC

Figure 1. Continued.

To confirm the effects on Mcl-1 in ruxolitinib-mediated apoptosis in CRC cells, Mcl-1 was overexpressed in LS411N and SW620 cells through cell transfection (Fig. 2C). Overexpression of Mcl-1 significantly decreased LS411N and SW620 sensitivity to ruxolitinib by inducing apoptosis
(Fig. 2D and E). These investigations suggested that ruxolitinib targeted Mcl-1 to trigger CRC cell apoptosis.

Involvement of pSTAT1 (S727) in the ruxolitinib-mediated decrease of Mcl-1. According to Timofeeva's report, 
G
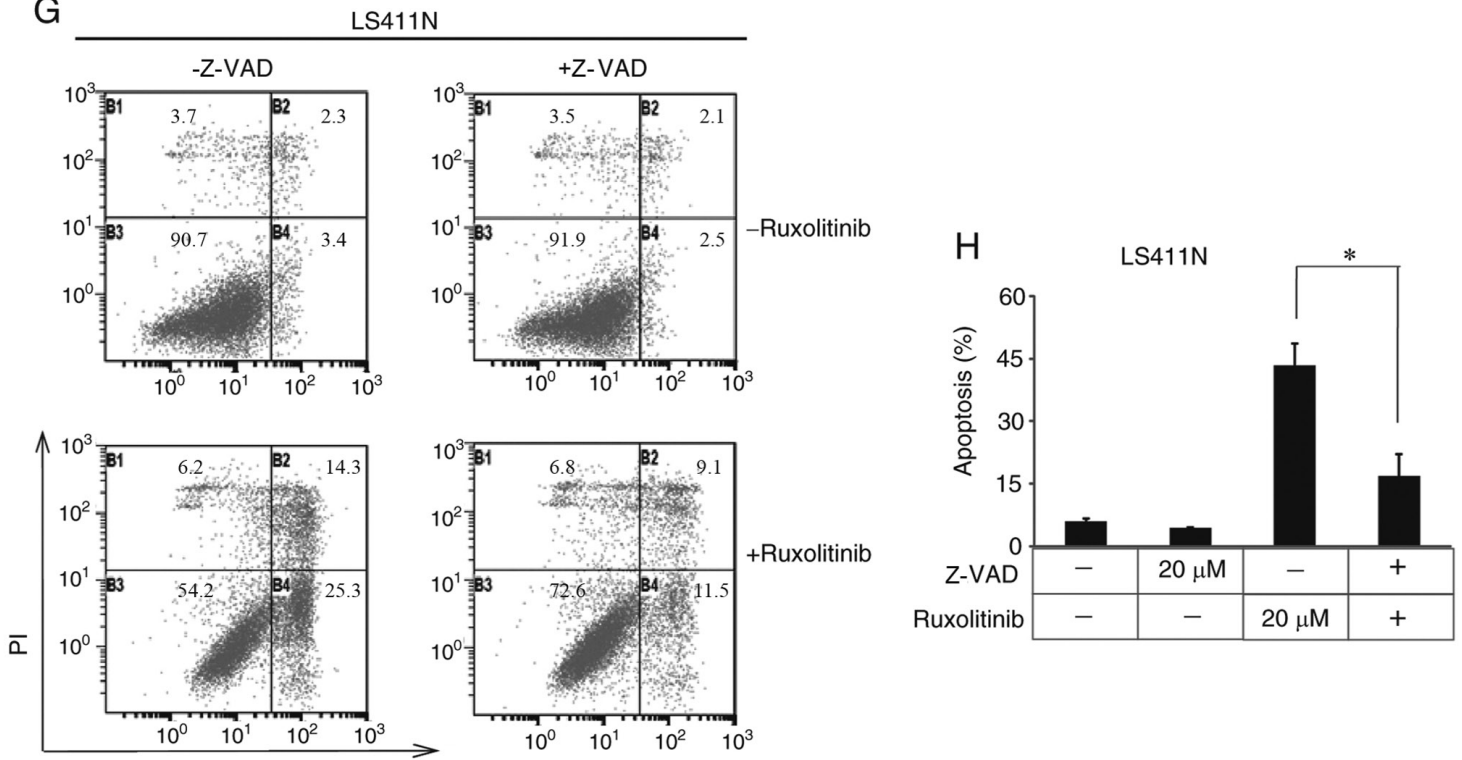

Annexin V-FITC

I
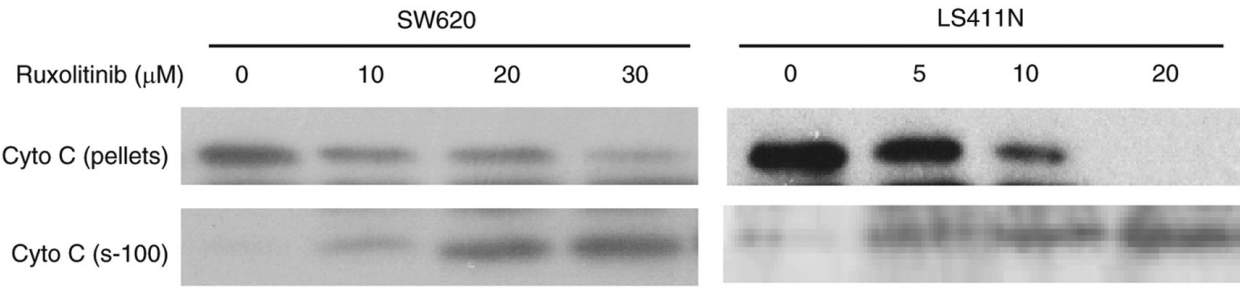

Cyto C (s-100)

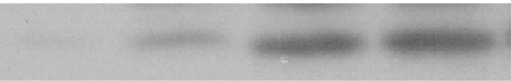

$\beta$-actin
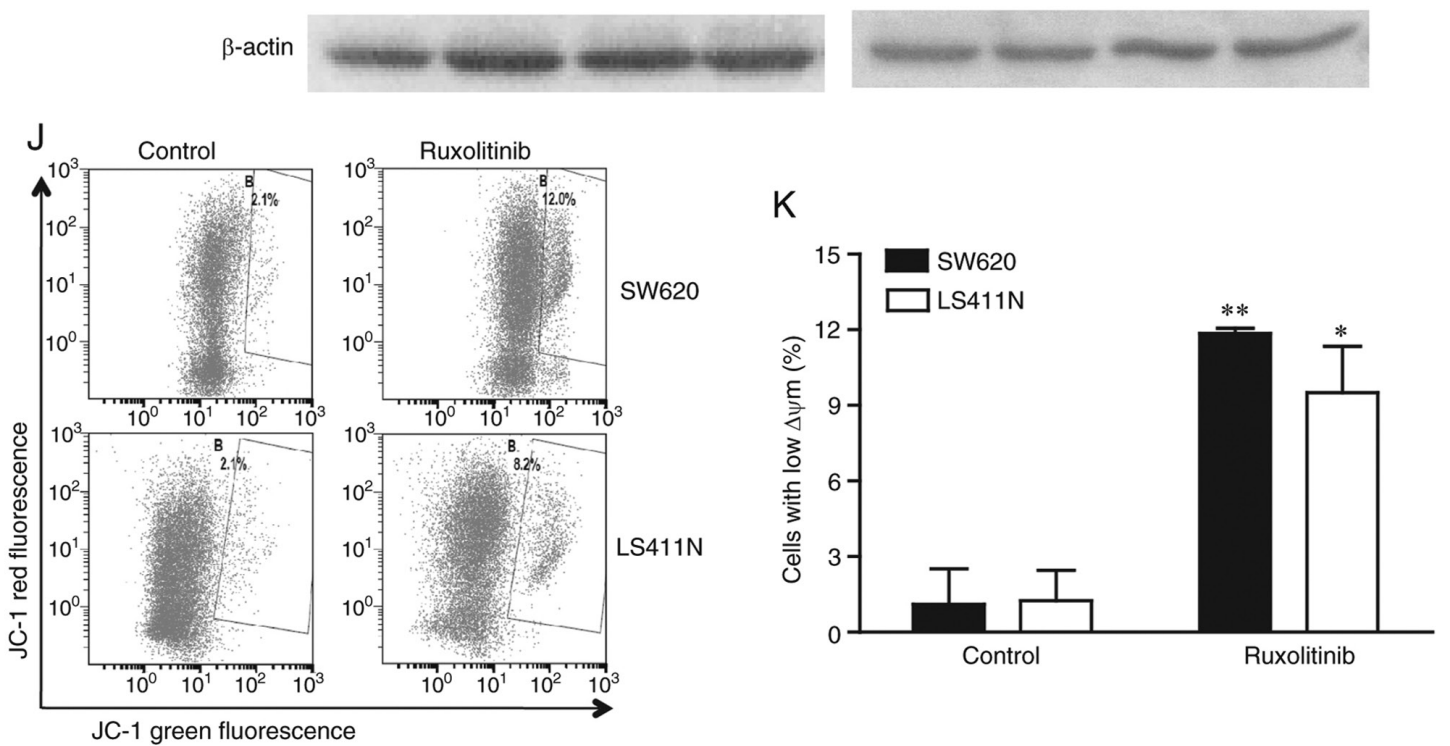

Figure 1. Ruxolitinib induces apoptosis in human colorectal cancer cells. (A) Cytotoxicity of ruxolitinib in LS411N, CT-26, SW620, DLD-1, HCT116 and SW480 cells was assessed using an MTT assay. (B) SW620 and LS411N cells were treated with indicated concentrations of ruxolitinib for $48 \mathrm{~h}$ and the apoptotic assay was performed. (C) Apoptotic cells were quantified and presented. ${ }^{*} \mathrm{P}<0.05$ or ${ }^{* * *} \mathrm{P}<0.01$ or ${ }^{* * * *} \mathrm{P}<0.001$ vs. control. (D) Caspase activation by ruxolitinib treatment. Cancer cells were cultured in the presence ruxolitinib for $48 \mathrm{~h}$, and then samples were analyzed using western blotting. (E) SW620 cells were cultured in the presence of ruxolitinib $(30 \mu \mathrm{M})$ in the absence or presence of Z-VAD-FMK for $48 \mathrm{~h}$ and the apoptotic assay was performed. (F) Apoptotic cells were quantified and presented. ${ }^{* *} \mathrm{P}<0.01$ vs. ruxolitinib only. (G) LS411N cells were cultured in the in the absence or presence of ruxolitinib (20 $\left.\mu \mathrm{M}\right)$ and Z-VAD-FMK for $48 \mathrm{~h}$, and then the apoptotic assay was performed. (H) Apoptotic cells were quantified and presented. "P<0.05 vs. ruxolitinib only. (I) SW620 and LS411N cells were treated with ruxolitinib for $48 \mathrm{~h}$. Cytosolic (s-100) and mitochondria-enriched fractions (pellets) were then prepared and analyzed using western blotting for Cyto C release. (J) Depolarization of mitochondrial membrane in ruxolitinib-treated SW620 and LS411N cells. Right-hand numbers correspond with the percentage of cell fraction with a depolarized mitochondrial membrane. (K) Depolarization of mitochondrial membrane in ruxolitinib-treated SW620 and LS411N cells was quantified and presented. ${ }^{*} \mathrm{P}<0.05,{ }^{* *} \mathrm{P}<0.01$ vs. control. Data are presented as the mean \pm standard deviation (n=3). Z-VAD, Z-VAD-FMK; Cyto C, cytochrome c.

serine-phosphorylated STAT1 is a pro-survival factor in Wilms' tumor pathogenesis (28). To study the inhibitory effects of ruxolitinib on STAT1 activity in CRC, the endogenous phosphorylation status of STAT1 in various CRC 
A
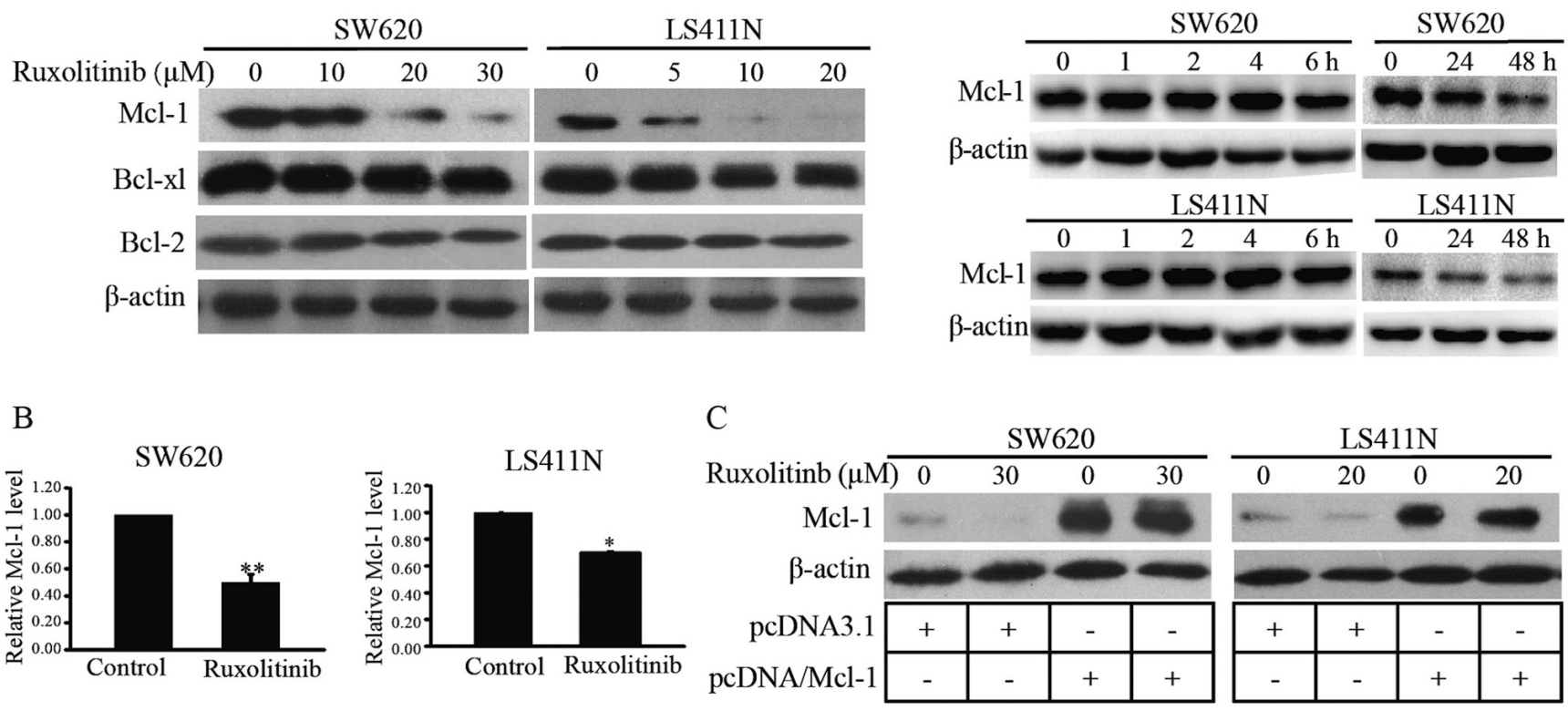

$\mathrm{D}$

SW620
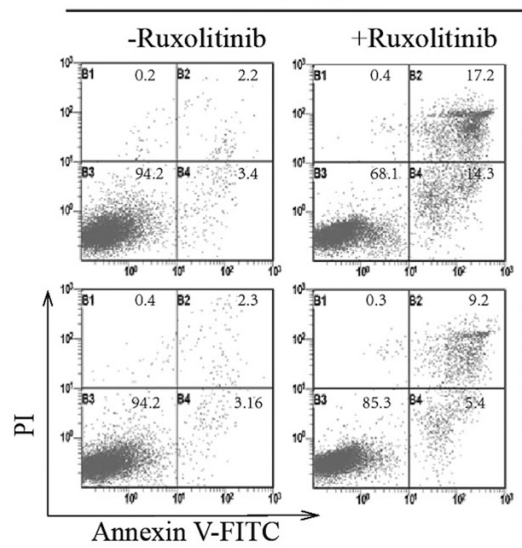

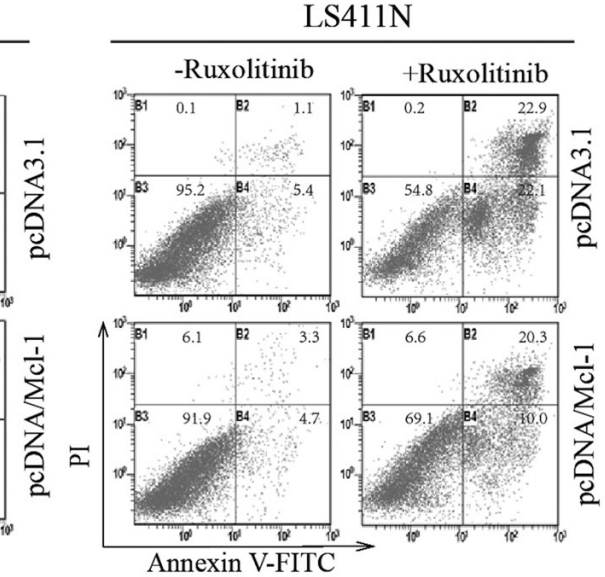

$\mathrm{E}$

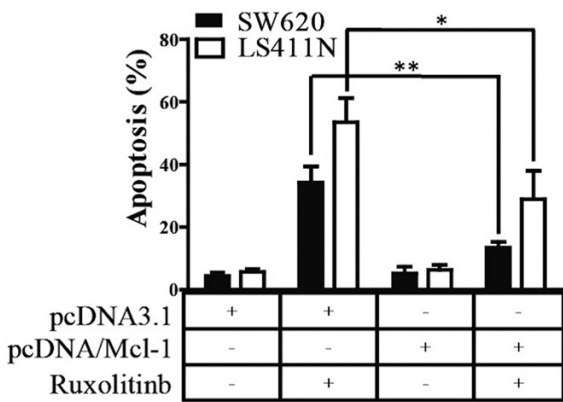

Figure 2. Molecular target of ruxolitinib is Mcl-1 in human colorectal cancer cells. (A) SW620 and LS411N cells were treated with ruxolitinib (30 and $20 \mu \mathrm{M}$, respectively) for $48 \mathrm{~h}$. Samples were then analyzed the Bcl-2 family proteins level using western blotting. SW620 and LS411N cells were treated with ruxolitinib for the indicated times. The expression of Bcl-2 family proteins were then analyzed using western blotting. (B) SW620 and LS411N cells were treated with ruxolitinib for $24 \mathrm{~h}$ and reverse transcription-quantitative PCR analysis was used to determine the Mcl-1 expression level. (C) SW620 and LS411N cells were transfected with a control vector or Mcl-1-expression vector. Then, cells were treated with the indicated concentration of ruxolitinib for $48 \mathrm{~h}$. Overexpression efficiency was analyzed using western blotting. (D) Flow cytometric analysis of cells treated as in (C). (E) Apoptosis was quantified and presented in the histogram. ${ }^{*} \mathrm{P}<0.05$ or ${ }^{* * *} \mathrm{P}<0.01$ vs. respective control. p, phosphorylated; Mcl-1, myeloid cell leukemia-1.

cell lines was examined using western blotting. As shown in Fig. 3A, ruxolitinib decreased JAK1, JAK2, STAT1 and pSTAT1 (S727) protein levels in a dose-dependent manner in LS411N and SW620 cells. Furthermore, different Mcl-1, STAT1 and pSTAT1 (S727) protein levels were found in the CRC cell lines (Fig. 3B); very low STAT1, pSTAT1 (S727) and high Mcl-1 were observed in HCT116 and SW480 cells, high STAT1, pSTAT1 (S727) and low Mcl-1 in DLD-1 cells and high STAT1, pSTAT1 (S727) and Mcl-1 were detected in LS411N and SW620 cells.

To validate the function of pSTAT1 (S727) in ruxolitinib-induced apoptosis in CRC cells, STAT1 was knocked down in LS411N and SW620 cells using transfection. As expected, western blot analysis indicated that the transfection of LS411N and SW620 cells with siRNA-STAT1 notably decreased STAT1 and pSTAT1 (S727) protein levels in the tumor cells (Fig. 3C). These data suggested that the ruxolitinib decreased the transcriptional level of Mcl-1. If ruxolitinib indeed induced apoptosis by decreasing transcriptional levels of Mcl-1, knockdown of STAT1 and pSTAT1 (S727) would diminish ruxolitinib-induced apoptosis in the tumor cells. Knockdown of STAT1 significantly decreased LS411N and SW620 cell sensitivity to ruxolitinib by neither inducing Mcl-1 protein levels or increasing apoptosis (Fig. 3C-F). These observations suggested that ruxolitinib may decrease Mcl-1 protein levels by inhibiting transcription factor pSTAT1 (S727), and, in turn, inducing CRC cell apoptosis.

Ruxolitinib disrupts the association of Bak with Mcl-1 and induces Bak activation. Bak has an important role in the intrinsic pathway of apoptosis (29). Mcl-1 can bind and thereby 
A

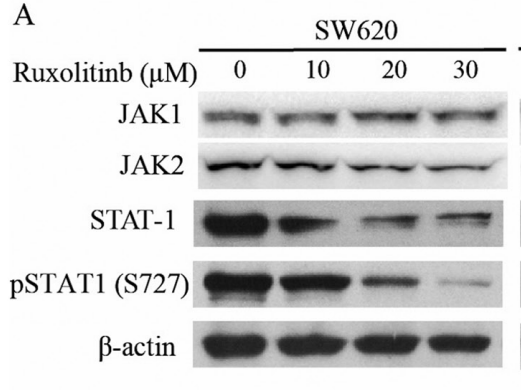

C
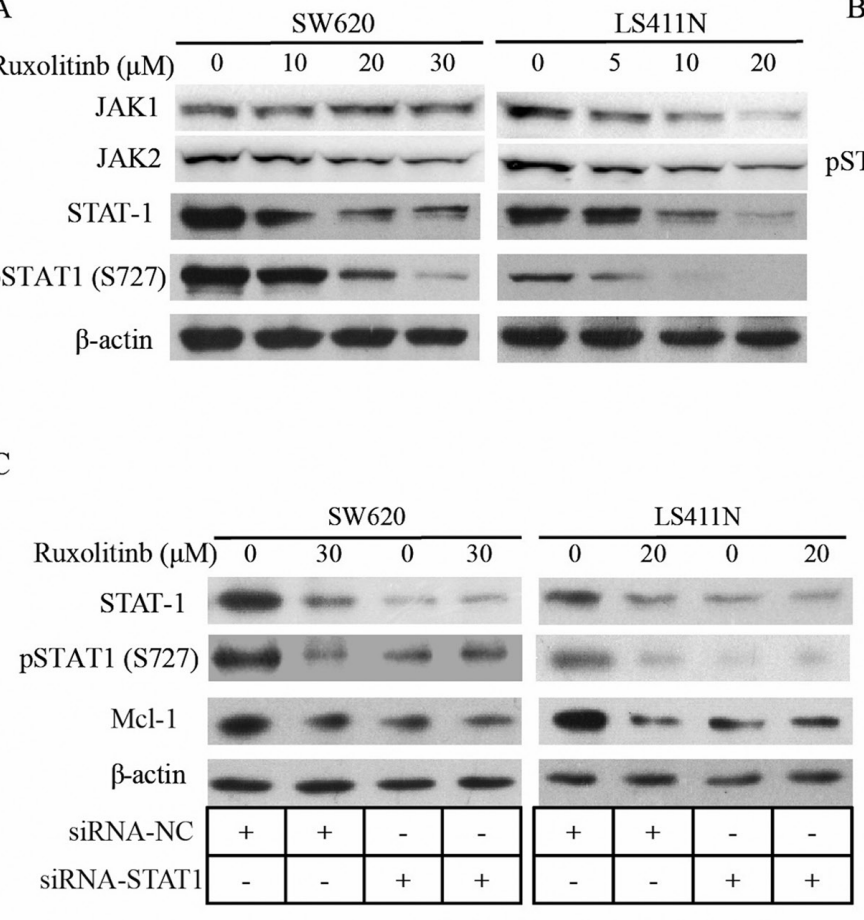

$\mathrm{D}$

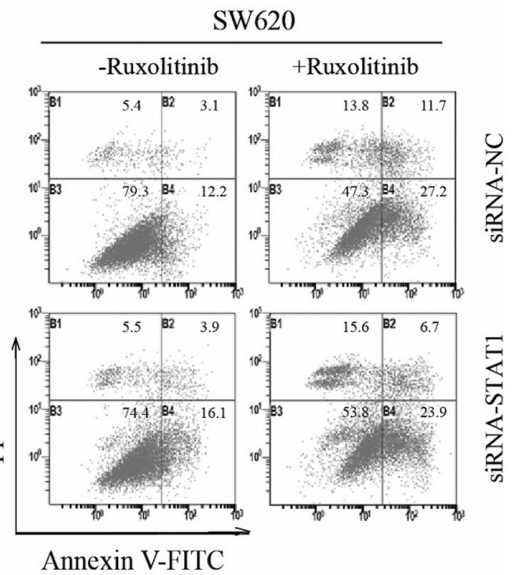

B

STAT-1

pSTAT1 (S727)

Mcl-1

$\beta$-actin

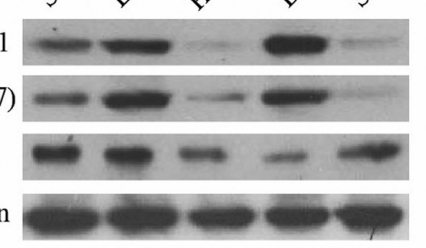

$\mathrm{E}$

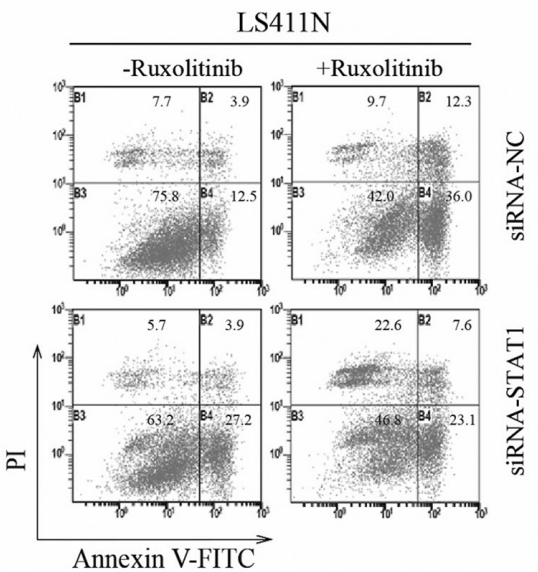

$\mathrm{F}$

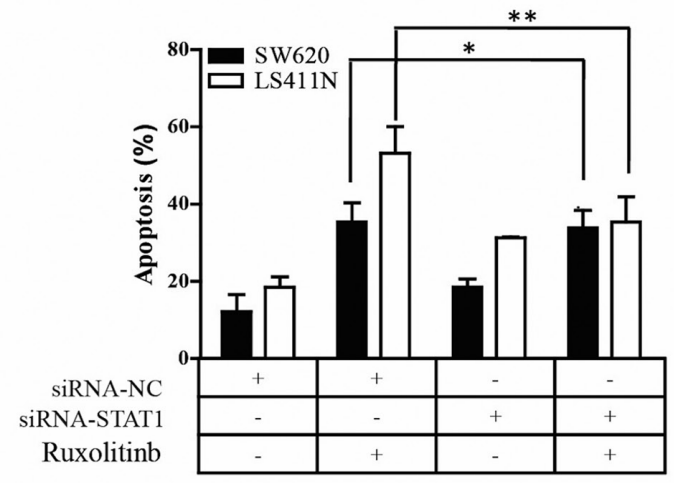

Figure 3. Serine-phosphorylated STAT1 mediated apoptotic resistance in human colorectal cancer cells. (A) SW620 and LS411N cells were cultured in the presence of various concentrations of ruxolitinib for $48 \mathrm{~h}$, and were then analyzed using western blotting. (B) Serine-phosphorylated STAT1, STAT1 and Mcl-1 in the indicated cell lines analyzed using western blotting. (C) SW620 and LS411N cells were transfected with siRNA-negative control or siRNA-STAT1 overnight. Cells were then treated with ruxolitinib for $48 \mathrm{~h}$. Silencing efficiency was analyzed using western blotting. (D and E) Flow cytometric analysis of cells treated as in (C). (F) Apoptotic cells were quantified. "P $<0.05$ or ${ }^{* *} \mathrm{P}<0.01$ vs. respective control. Mcl-1, myeloid cell leukemia-1; p, phosphorylated; si, small interfering; $\mathrm{NC}$, negative control.

inactivate pro-apoptotic Bak (30). Based on this connection between Mcl-1 and Bak, it was hypothesized that the inhibition of Mcl-1 by ruxolitinib might release Bak, which, in turn, may induce apoptosis. To verify this hypothesis, the effect of ruxolitinib on Bak activation and combination of Bak with Mcl-1 was examined. Western blot analysis revealed that ruxolitinib treatment increased total Bak protein levels (Fig. 4A). In addition, the Co-IP analysis indicated that Mcl-1 bound Bak in LS411N and SW620 cells, and ruxolitinib treatment decreased this binding (Fig. 4B). Furthermore, an increase in Bak conformational change was also observed after ruxolitinib treatment (Fig. 4C).
To validate the aforementioned findings, STAT1 was knocked down in LS411N and SW620 cells, and Bak activation was analyzed using flow cytometry. Bak activation is related with a conformational change, which can be detected by antibodies only recognizing the active protein conformer (31). Knockdown of STAT1 blocked ruxolitinib-induced Bak activation (Fig. 4D). In short, these data suggested that ruxolitinib decreased Mcl-1 to release Bak, whose activation could trigger apoptosis.

Oral administration of ruxolitinib significantly suppresses CRC tumor growth in vivo. To further determine the anticancer efficacy of ruxolitinib treatment in vivo, a xenograft model 
A

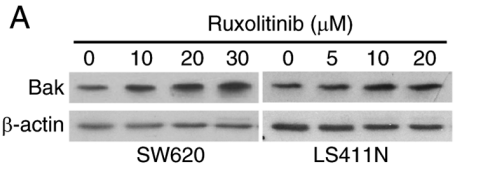

C
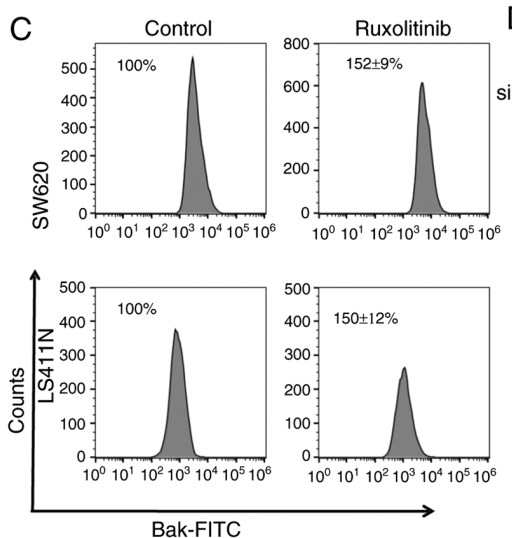

B
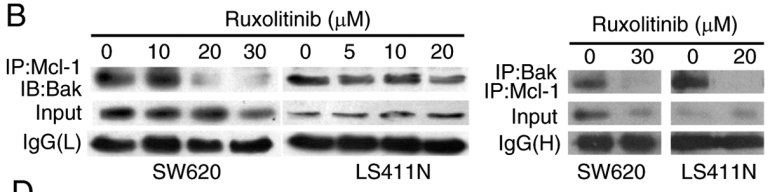

$\mathrm{D}$

\begin{tabular}{|r|c|c|c|c|}
\cline { 2 - 5 } SiRNA-NC & + & + & - & - \\
\cline { 2 - 5 } SiRNA-STAT1 & - & - & + & + \\
\cline { 2 - 5 } Ruxolitinb & - & + & - & + \\
\cline { 2 - 5 } & & &
\end{tabular}
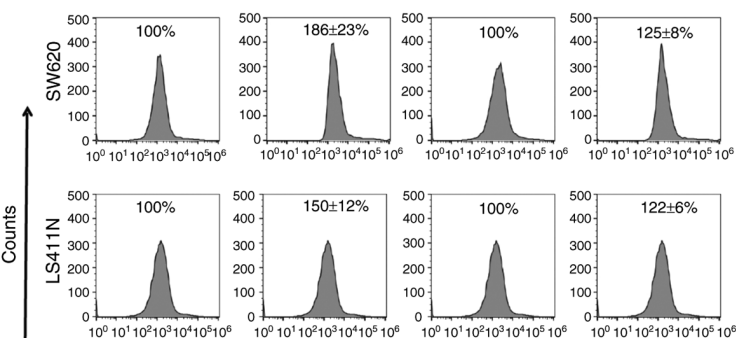

\begin{tabular}{|l|l|}
400 \\
400 \\
300 \\
200 \\
100
\end{tabular}
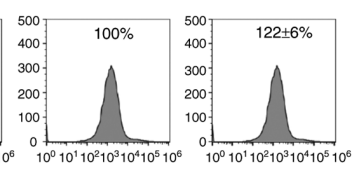

Bak-FITC

Figure 4. Ruxolitinib induced Bak activation in human colorectal cancer cells. (A) BAK was increased by ruxolitinib treatment. (B) Mcl-1 and Bak associations were examined by reciprocal immunoprecipitation and western blotting. (C) SW620 and LS411N cells were treated with ruxolitinib for $48 \mathrm{~h}$. After treatment, cells were stained with anti-conformationally changed Bak antibody, and subjected to flow cytometry. (D) SW620 and LS411N cells were transfected with siRNA-negative control or siRNA-STAT1 overnight. Cells were then treated with ruxolitinib for $48 \mathrm{~h}$. After treatment, the cells were then stained with anti-conformationally changed Bak antibody, and subjected to flow cytometry. Mcl-1, myeloid cell leukemia-1; si, small interfering.

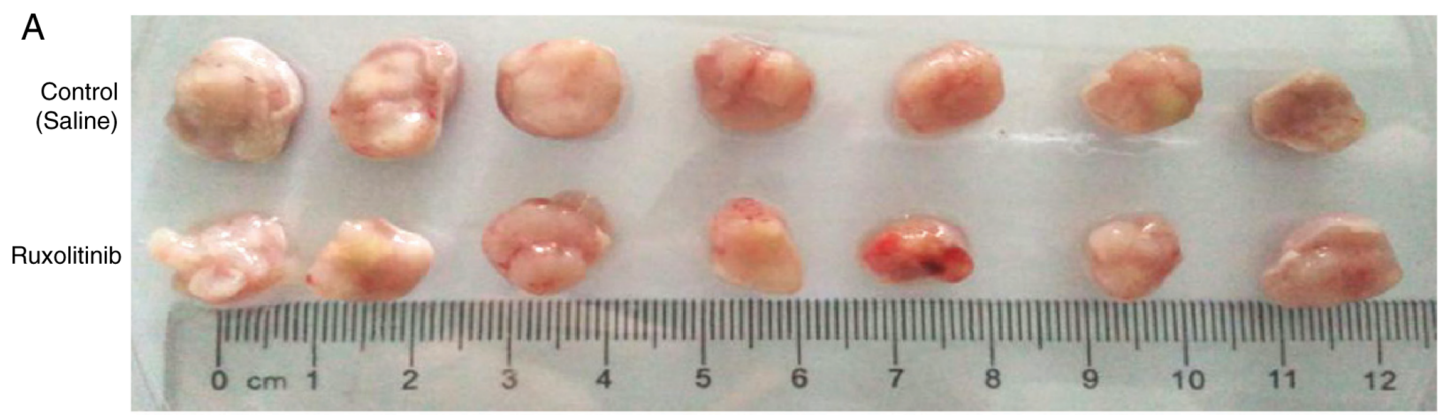

$\mathrm{B}$

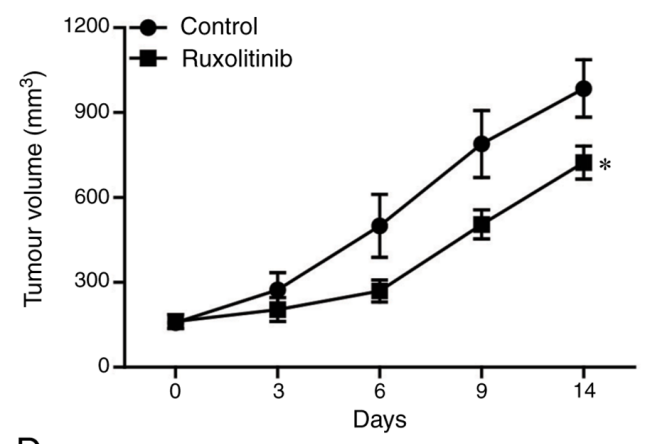

D

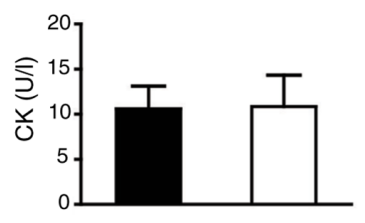

C

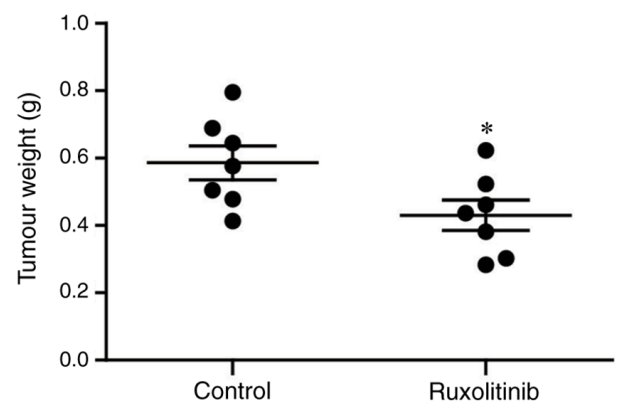

$\mathrm{E}$

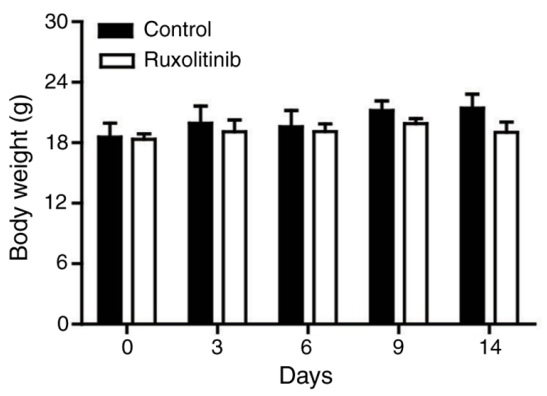

Figure 5. Ruxolitinib suppresses LS411N xenograft growth in vivo. (A) LS411N cells were injected to nude mice. Tumor-bearing mice were then treated either with saline or ruxolitinib. (B) Tumor size was measured. (C) Tumor weight of two groups. (D) Mouse serum toxicity profiles of ruxolitinib. Measurements were performed 14 days after ruxolitinib injection. CK, creatine kinase; ALT, alanine aminotransferase. (E) Body weights of the mice. Data are presented by mean $\pm \mathrm{SD}, \mathrm{n}=7$. ${ }^{*} \mathrm{P}<0.05$ vs. respective control. 
$\begin{array}{lllllllllllllll}\text { A } & \text { C1 } & \text { N1 } & \text { C2 } & \text { N2 } & \text { C3 } & \text { N3 } & \text { C4 } & \text { N4 } & \text { C5 } & \text { N5 } & \text { C6 } & \text { N6 }\end{array}$

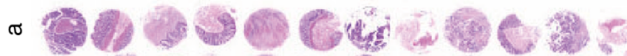

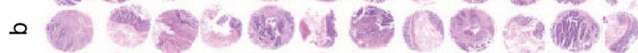

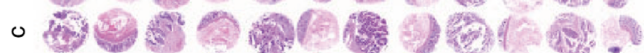

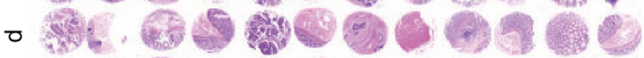

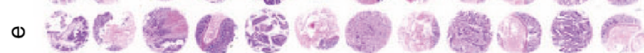

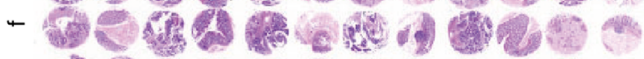

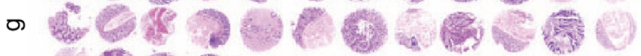

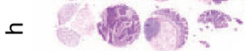
$2000 \mu \mathrm{m}$ HE

C C6c

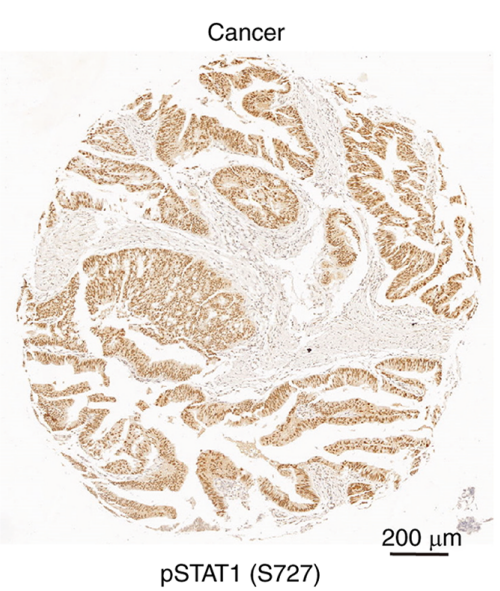

D

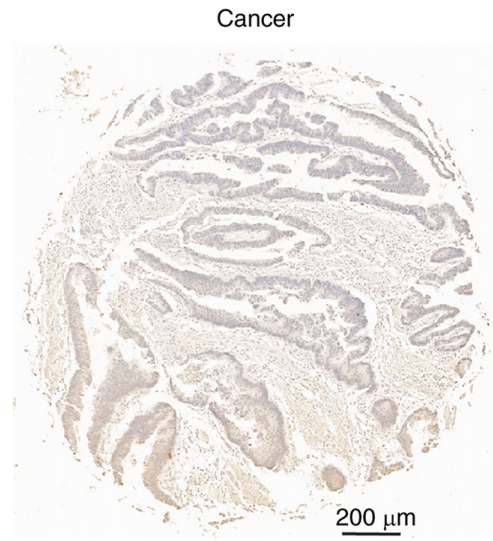

PSTAT1 (S727)
B $\begin{array}{lllllllllllll}\text { C1 } & \text { N1 } & \text { C2 } & \text { N2 } & \text { C3 } & \text { N3 } & \text { C4 } & \text { N4 } 4 & \text { C5 } & \text { N5 } & \text { C6 } & \text { N6 }\end{array}$

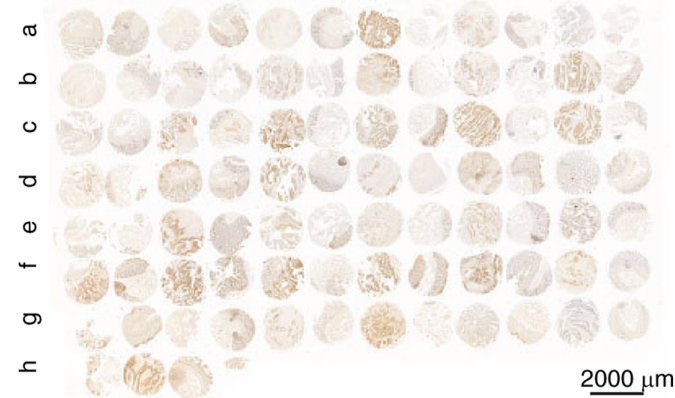

pSTAT1 (S727)

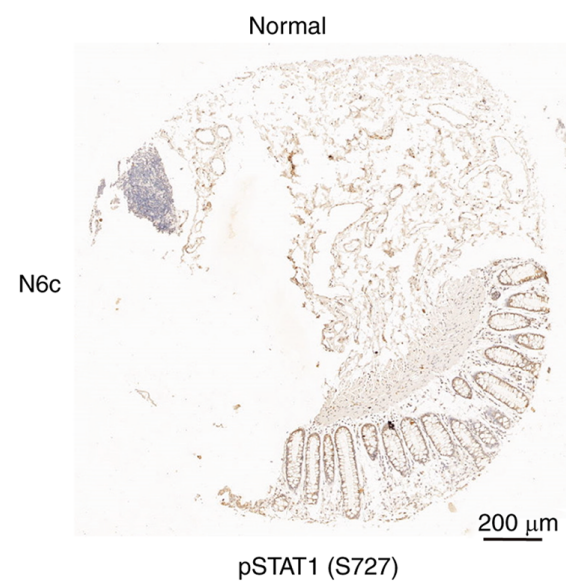

N6g

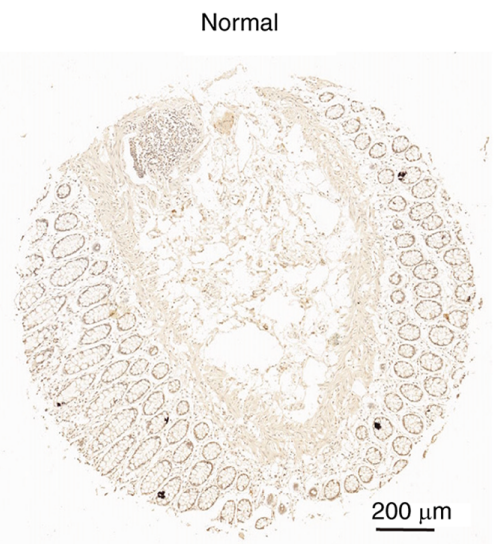

pSTAT1 (S727)

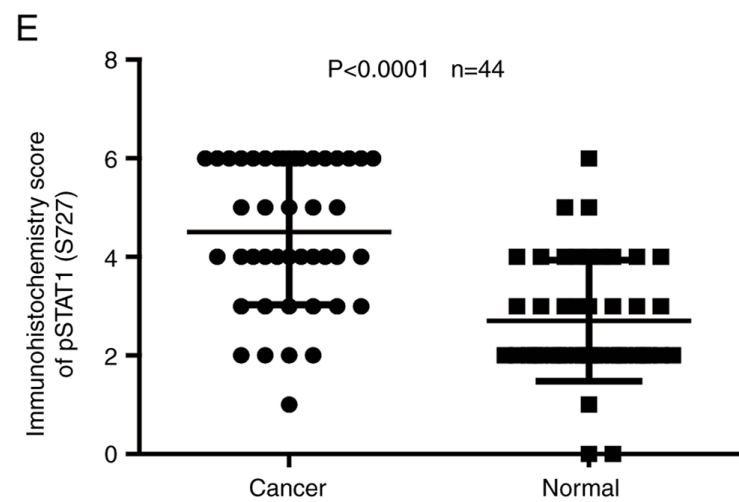

Figure 6. Expression of pSTAT1 (S727) protein level in patients with CRC patients. (A) Hematoxylin and eosin-stained sections of tumors and paired normal tissues from patients with CRC. (B) Immunohistochemistry for pSTAT1 (S727) in normal colon and primary tumor. Brown color indicates pSTAT1 (S727) protein levels, with counterstaining by hematoxylin in blue. (C) Representative images of positive pSTAT1 (S727)-stained samples. (D) Representative images of low pSTAT1 (S727)-stained samples. (E) Quantification of immunohistochemistry score of pSTAT1 (S727) in tumors and paired normal tissues from patients with CRC. p, phosphorylated; CRC, colorectal cancer; N, normal tissue; C, colorectal cancer. 


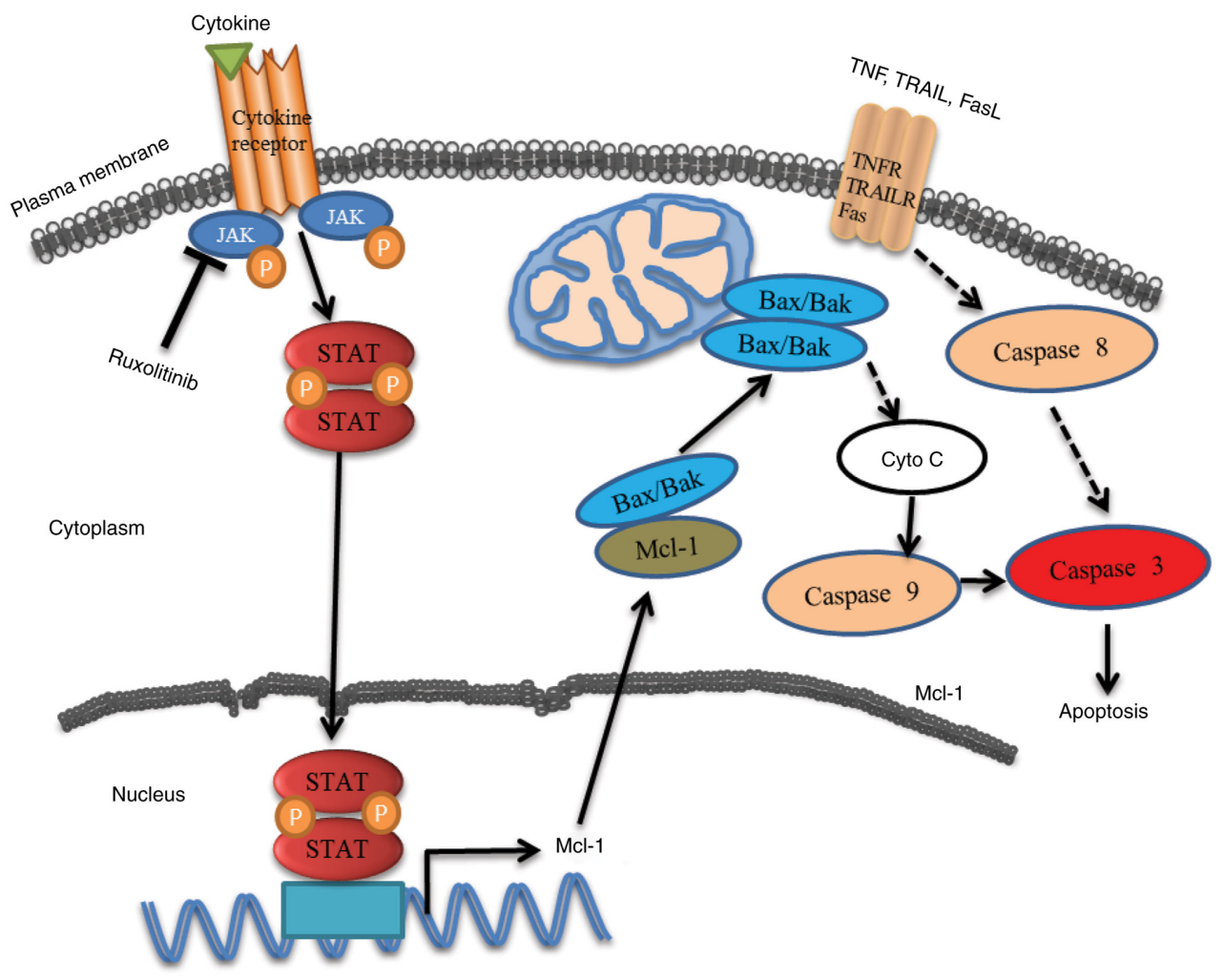

Figure 7. Proposed model for the relationship between JAK1/2-STAT1-Mcl-1 signaling and ruxolitinib-induced apoptosis. JAK, Janus kinase; p, phosphorylated; Cyto C, cytochrome c; Mcl-1, myeloid cell leukemia-1.

was constructed using LS411N cells. As shown in Fig. 5A-C, significantly lower tumor volume and weight were observed in mice treated with ruxolitinib compared with the control group (both $\mathrm{P}<0.05$ ).

Furthermore, serum creatine kinase and alanine aminotransferase levels were analyzed to determine ruxolitinib liver toxicity. The two enzymes were unchanged by ruxolitinib (Fig. 5D). Meanwhile, no significant differences in animal body weight were found between the treatment and control group (Fig. 5E), which further suggested that ruxolitinib was not toxic to mice. Taken together, these data showed that ruxolitinib could effectively suppress LS411N xenograft growth in vivo without inducing liver toxicity.

Expression of pSTAT1 (S727) protein level in patients with $C R C$. To determine the expression levels of pSTAT1 (S727) in human colon carcinoma, 44 pairs of human colon carcinoma and adjacent normal tissue specimens were examined using immunohistochemical staining. The results showed that hematoxylin and eosin staining (Servicebio Company, Hangzhou) (Fig. 6A) and pSTAT1 (S727) staining (Fig. 6B) images from tumor samples and paired normal tissues from patients with CRC. Furthermore, Fig. 6C and D shows the representative pSTAT1 (S727) high and low staining images of tumors and paired normal tissues. In total, 36.4\% (16/44) of patients showed the positive expression of pSTAT1 (S727) in normal tissues; however, 81.8\% (36/44) of patients showed positive pSTAT1 (S727) in paired tumor tissues. The staining scores indicated that the expression levels of pSTAT1 (S727) were significantly higher in tumor tissues compared with those in matched normal tissues $(\mathrm{P}<0.0001$; Fig. 6E). STAT1 phosphorylation on S727 in most CRC specimens illustrated that this modification might be involved in the pathogenesis of this tumor.

\section{Discussion}

JAK, a kind of tyrosine kinase, serves key roles in the differentiation, proliferation and cell death of both normal and cancer cells (32). JAKs are activated by growth factor receptors, cytokines, such as IL- 6 and IFN- $\gamma$, and downstream signaling proteins, such as STATs (33). STAT1 is part of the JAK/STAT signaling cascade. STAT1 modulates various cellular processes, such as antimicrobial activities, cell proliferation and cell death (34). STAT1 is activated by different cytokines, including type I-III interferons, interleukin (IL)-21 and IL-27, and is transiently and tightly regulated (35). Tyrosine 701 phosphorylation on STAT1 through JAKs leads to its activation and nuclear translocation. Meanwhile, serine 727 phosphorylation is required for transcriptional activation in response to cellular stress (36). Yet, the role of STAT1 in carcinogenesis is still not well defined. Some studies have suggested that STAT1 may suppress tumorigenesis and/or metastasis in various types of cancer, including hepatocellular carcinoma, esophageal cancer, CRC, pancreatic cancer and metastatic melanoma (37-41). In addition, other studies have suggested that STAT1 promotes tumor growth by inhibiting tumor immune surveillance and by promoting tumor resistance against chemotherapy and irradiation. For example, activation of STAT1 by the cancer-up-regulated gene 2 enhances metastasis and drug resistance in colon cancer cells (42-44). Taken together, these data suggest 
that JAK-STAT activity is likely cancer type-dependent, especially in CRC.

The present study examined the inhibitory effects of ruxolitinib on human CRC cells. It was demonstrated that ruxolitinib inhibited CRC cell viability through the induction of apoptosis and by activating both intrinsic and extrinsic pathways. Furthermore, Mcl-1 was identified as the downstream molecular target of ruxolitinib, which inhibited JAK1 and/or JAK2. It was also reported that $\mathrm{Mcl}-1$ is regulated by transcription factor pSTAT1 (S727). Ruxolitinib decreased Mcl-1 mRNA without affecting mRNA stability, which in turn enabled Bak to trigger CRC apoptosis. In addition, the in vivo experiment suggested that ruxolitinib was effective in the suppression of CRC tumor growth without inducing liver toxicity. Moreover, an analysis of 44 matched pairs of normal human colorectal tissues and CRC tissues revealed that pSTAT1 (S727) expression was significantly higher in the tumor tissues compared with in the adjacent normal colorectal tissues.

Mcl-1 is a member of the antiapoptotic Bcl-2 family of proteins that represses apoptosis through binding with pro-apoptotic proteins, such as Bak, that inhibit its activation and subsequently prevent cytochrome c release from mitochondria (45). Mcl-1 is a highly regulated protein with a short half-life; it is induced by a broad range of survival signals, such as AKT and ERK signals, and is quickly downregulated during apoptosis (14). Mcl-1 can be regulated at both the transcriptional and translational levels. Transcriptionally, Mcl-1 expression can be induced by various cytokines and signaling pathways, including PI3K/AKT and JAK/STAT pathways $(46,47)$. Previous studies have highlighted the importance of microRNAs in Mcl-1 regulation at the translational level (48-50). In addition, Mcl-1 is highly expressed in human cancer cell lines, including breast, colon, lung, ovarian, prostate and renal cancer, and melanoma (51). Aberrant expression of Mcl-1 is an important genomic change present in multiple cancer types, a number of which become dependent upon this protein for cell survival and resistance to chemotherapy. For example, our previous study revealed that Mcl-1, which induces proteasome-dependent degradation by imperatorin, is an important factor in the mechanism of liver cancer pathophysiology that can also cause resistance to doxorubicin-based therapy (14). It is known that, transcriptionally, Mcl-1 expression can be regulated by STAT-3 (46). Downregulation of STAT-3 induces a decrease in Mcl-1 transcription, indicating that STAT-3 inhibition could play a significant role in the induction of apoptosis (52). Timofeeva et al reported that serine-phosphorylated STAT1 promotes cancer cell survival through downregulation of Mcl-1 expression (28). Some reports have reported a correlation between STAT-1 or STAT-3 serine phosphorylation with increased DNA-binding ability; however, sufficient evidence for this observation has not been provided $(36,53,54)$. In the present study, Mcl-1 was downregulated in SW620 and LS411N cells under ruxolitinib treatment when transcription factor serine-phosphorylated STAT1 was decreased. Moreover, an overexpression of Mcl-1 effectively reduced LS411N and SW620 cells sensitivity to ruxolitinib by inducing apoptosis. Notably, knockdown of STAT1 induced apoptosis in LS411N and
SW620 cells and also significantly decreased the sensitivity of these cells to ruxolitinib-induced apoptosis. Therefore, these observations suggested that serine-phosphorylated STAT1 can maintain the expression of Mcl-1 in LS411N and SW620 cells, thus enabling the survival of tumor cells under the same conditions.

In summary, the results suggested ruxolitinib that inhibited JAK1 and/or JAK2 and could decrease STAT1 S727 phosphorylation. In addition, it was demonstrated that Mcl-1 is regulated by the transcription factor pSTAT1 (S727). Ruxolitinib decreased Mcl-1 mRNA levels without affecting mRNA stability, which in turn enabled Bak to trigger caspase-dependent apoptosis in human CRC cells (Fig. 7). The present study identified JAK1/2-STAT1-Mcl-1 as important factors and potential molecular therapeutic targets in human CRC. However, based on the current results, the JAK1/2-STAT1-Mcl-1 axis is insufficient to function as a biomarker for CRC prognosis. Prospective studies will aim to investigate the mechanisms of pSTAT1 (S727) in CRC and the interaction of relevant factors.

\section{Acknowledgements}

Not applicable.

\section{Funding}

The present study was funded by the National Natural Science Foundation of China (grant nos. 31570811, 81673813, 81502603 and 81903641) the Zhejiang Provincial Natural Science Foundation of China (grant nos. LQ18H160016, LGF18H160016 and LY20H160039), the International Cooperation Fund from Science Technology Department of Zhejiang Province (grant no. LGJ19H160001) and the Medicine \& Health Science Fund of Zhejiang Province (grant no. 2018RC021).

\section{Availability of data and materials}

The datasets used and/or analyzed during the present study are available from the corresponding author upon reasonable request.

\section{Authors' contributions}

XL, WC and FL drafted the initial manuscript and confirmed the authenticity of all the raw data. XL, ZW, WC and FL participated in the collection, analysis and interpretation of data. XL, ZW, SZ, QY, WC and FL performed the experiments, and collected and analyzed the data. WC and FL conceived of the study and participated in its design and coordination and provided important inputs for the manuscript. All authors have read and approved the final manuscript.

\section{Ethics approval and consent to participate}

The present study was approved by the Medical Ethics Committee of Zhejiang Cancer Hospital (Hangzhou, China; approval no. ZJU20180108020). All patients provided written informed consent prior to the study start. 


\section{Patient consent for publication}

Not applicable.

\section{Competing interests}

They authors declare that they have no competing interests.

\section{References}

1. Siegel R, Naishadham D and Jemal A: Cancer statistics, 2013 CA Cancer J Clin 63: 11-30, 2013.

2. Chen W, Zheng R, Baade PD, Zhang S, Zeng H, Bray F, Jemal A, Yu XQ and He J: Cancer statistics in China, 2015. CA Cancer J Clin 66: 115-132, 2016.

3. Li X, Zhou Y, Luo Z, Gu Y, Chen Y, Yang C, Wang J, Xiao S, Sun Q, Qian M and Zhao G: The impact of screening on the survival of colorectal cancer in Shanghai, China: A population based study. BMC Public Health 19: 1016, 2019.

4. Meads MB, Gatenby RA and Dalton WS: Environment-mediated drug resistance: A major contributor to minimal residual disease. Nat Rev Cancer 9: 665-674, 2009.

5. Zhao H, Zhang N, Ho V, Ding M, He W, Niu J, Yang M, Du XL, Zorzi D, Chavez-MacGregor M and Giordano SH: Adherence to treatment guidelines and survival for older patients with stage II or III colon cancer in Texas from 2001 through 2011. Cancer 124 679-687, 2018

6. Lieberman DA, Rex DK, Winawer SJ, Giardiello FM, Johnson DA and Levin TR: Guidelines for colonoscopy surveillance after screening and polypectomy: A consensus update by the US multi-society task force on colorectal cancer. Gastroenterology 143: 844-857, 2012.

7. Harada T, Yamamoto E, Yamano HO, Aoki H, Matsushita HO, Yoshikawa K, Takagi R, Harada E, Tanaka Y, Yoshida Y, et al: Surface microstructures are associated with mutational intratumoral heterogeneity in colorectal tumors. J Gastroenterol 53: 1241-1252, 2018

8. Rawlings JS, Rosler KM and Harrison DA: The JAK/STAT signaling pathway. J Cell Sci 117: 1281-1283, 2004.

9. O'Shea JJ, Schwartz DM, Villarino AV, Gadina M, McInnes IB and Laurence A: The JAK-STAT pathway: Impact on human disease and therapeutic intervention. Annu Rev Med 66: 311-328, 2015.

10. Sansone $\mathrm{P}$ and Bromberg J: Targeting the interleukin-6/Jak/stat pathway in human malignancies. J Clin Oncol 30: 1005-1014, 2012.

11. Spano JP, Milano G, Rixe C and Fagard R: JAK/STAT signalling pathway in colorectal cancer: A new biological target with therapeutic implications. Eur J Cancer 42: 2668-2670, 2006.

12. Wang SW and Sun YM: The IL-6/JAK/STAT3 pathway: Potential therapeutic strategies in treating colorectal cancer (Review). Int J Oncol 44: 1032-1040, 2014.

13. Yamaguchi R, Lartigue L and Perkins G: Targeting Mcl-1 and other Bcl-2 family member proteins in cancer therapy. Pharmacol Ther 195: 13-20, 2019.

14. Li X, Zeng X, Sun J, Li H, Wu P, Fung KP and Liu F: Imperatorin induces Mcl-1 degradation to cooperatively trigger Bax translocation and Bak activation to suppress drug-resistant human hepatoma. Cancer Lett 348: 146-155, 2014.

15. Craig RW: MCL1 provides a window on the role of the BCL2 family in cell proliferation, differentiation and tumorigenesis. Leukemia 16: 444-454, 2002.

16. Gomez-Bougie P, Halliez M, Moreau P, Pellat-Deceunynck C and Amiot M: Repression of Mcl-1 and disruption of the Mcl-1/Bak interaction in myeloma cells couple ER stress to mitochondrial apoptosis. Cancer Lett 383: 204-211, 2016.

17. Verstovsek S, Kantarjian H, Mesa RA, Pardanani AD, Cortes-Franco J, Thomas DA, Estrov Z, Fridman JS, Bradley EC, Erickson-Viitanen S, et al: Safety and efficacy of INCB018424, a JAK1 and JAK2 inhibitor, in myelofibrosis. N Engl J Med 363: $1117-1127,2010$.

18. Shilling AD, Nedza FM, Emm T, Diamond S, McKeever E, Punwani N, Williams W, Arvanitis A, Galya LG, Li M, et al Metabolism, excretion, and pharmacokinetics of [14C] INCB018424, a selective Janus tyrosine kinase $1 / 2$ inhibitor, in humans. Drug Metab Dispos 38: 2023-2031, 2010
19. Stover DG, Gil Del Alcazar CR, Brock J, Guo H, Overmoyer B, Balko J, Xu Q, Bardia A, Tolaney SM, Gelman R, et al: Phase II study of ruxolitinib, a selective JAK1/2 inhibitor, in patients with metastatic triple-negative breast cancer. NPJ Breast Cancer 4: 10, 2018.

20. Galvez Acosta S and Javalera Rincon M: Ruxolitinib as first-line therapy in secondary hemophagocytic lymphohistiocytosis and HIV infection. Int J Hematol 112: 418-421, 2020.

21. Harrison C, Kiladjian JJ, Al-Ali HK, Gisslinger H, Waltzman R, Stalbovskaya V, McQuitty M, Hunter DS, Levy R, Knoops L, et al: JAK inhibition with ruxolitinib versus best available therapy for myelofibrosis. N Engl J Med 366: 787-798, 2012.

22. Schonberg K, RudolphJ,VonnahmeM,Parampalli YajnanarayanaS, Cornez I, Hejazi M, Manser AR, Uhrberg M, Verbeek W, Koschmieder S, et al: JAK inhibition impairs NK cell function in myeloproliferative neoplasms. Cancer Res 75: 2187-2199, 2015.

23. Klatte $\mathrm{M}$ and Bauer P: Accurate real-time reverse transcription quantitative PCR. Methods Mol Biol 479: 61-77, 2009.

24. Li X, Wu J, Zhang X and Chen W: Glutathione reductase-mediated thiol oxidative stress suppresses metastasis of murine melanoma cells. Free Radic Biol Med 129: 256-267, 2018.

25. Du H, Chen L, Luo F, Chen X, Li Y and Cheng Q: Beclin-1 expression is associated with prognosis in a Bcl-2-dependent manner in non-small cell lung cancer. Oncol Lett 20: 9, 2020.

26. Yuan B, Hao J, Zhang Q, Wang Y and Zhu Y: Role of Bcl-2 on drug resistance in breast cancer polyploidy-induced spindle poisons. Oncol Lett 19: 1701-1710, 2020.

27. Zhou Y, Zhou Y, Yang M, Wang K, Liu Y, Zhang M, Yang Y, Jin C, Wang R and Hu R: Digoxin sensitizes gemcitabine-resistant pancreatic cancer cells to gemcitabine via inhibiting Nrf2 signaling pathway. Redox Biol 22: 101131, 2019.

28. Timofeeva OA, Plisov S, Evseev AA, Peng S, Jose-Kampfner M, Lovvorn HN, Dome JS and Perantoni AO: Serine-phosphorylated STAT1 is a prosurvival factor in Wilms' tumor pathogenesis. Oncogene 25: 7555-7564, 2006.

29. Reed JC: Proapoptotic multidomain Bcl-2/Bax-family proteins: Mechanisms, physiological roles, and therapeutic opportunities. Cell Death Differ 13: 1378-1386, 2006.

30. Cuconati A, Mukherjee C, Perez D and White E: DNA damage response and MCL-1 destruction initiate apoptosis in adenovirus-infected cells. Genes Dev 17: 2922-2932, 2003.

31. Chen S, Dai Y, Harada H, Dent P and Grant S: Mcl-1 down-regulation potentiates ABT-737 lethality by cooperatively inducing Bak activation and Bax translocation. Cancer Res 67: 782-791, 2007.

32. Rane SG and Reddy EP: Janus kinases: Components of multiple signaling pathways. Oncogene 19: 5662-5679, 2000.

33. Turkson J and Jove R: STAT proteins: Novel molecular targets for cancer drug discovery. Oncogene 19: 6613-6626, 2000.

34. Meissl K, Macho-Maschler S, Muller M and Strobl B: The good and the bad faces of STAT1 in solid tumours. Cytokine 89: 12-20, 2017.

35. Boisson-Dupuis S, Kong XF, Okada S, Cypowyj S, Puel A, Abel L and Casanova JL: Inborn errors of human STAT1: Allelic heterogeneity governs the diversity of immunological and infectious phenotypes. Curr Opin Immunol 24: 364-378, 2012.

36. Decker T and Kovarik P: Serine phosphorylation of STATs. Oncogene 19: 2628-2637, 2000.

37. Chen J, Wang H, Wang J, Huang S and Zhang W: STAT1 inhibits human hepatocellular carcinoma cell growth through induction of p53 and Fbxw7. Cancer Cell Int 15: 111, 2015.

38. Zhang Y, Molavi O, Su M and Lai R: The clinical and biological significance of STAT1 in esophageal squamous cell carcinoma. BMC Cancer 14: 791, 2014.

39. Zhang X, Li X, Tan F, Yu N and Pei H: STAT1 inhibits MiR-181a expression to suppress colorectal cancer cell proliferation through PTEN/Akt. J Cell Biochem 118: 3435-3443, 2017.

40. Sun Y, Yang S, Sun N and Chen J: Differential expression of STAT1 and $\mathrm{p} 21$ proteins predicts pancreatic cancer progression and prognosis. Pancreas 43: 619-623, 2014.

41. Osborn JL and Greer SF: Metastatic melanoma cells evade immune detection by silencing STAT1. Int J Mol Sci 16: 4343-4361, 2015.

42. Zhang N, Li F, Gao J, Zhang S and Wang Q: Osteopontin accelerates the development and metastasis of bladder cancer via activating JAK1/STAT1 pathway. Genes Genomics 42: 467-475, 2020. 
43. Wu J, Gao F, Xu T, Li J, Hu Z, Wang C, Long Y, He X, Deng X, Ren D, et al: CLDN1 induces autophagy to promote proliferation and metastasis of esophageal squamous carcinoma through AMPK/STAT1/ULK1 signaling. J Cell Physiol 235: 2245-2259, 2020.

44. Malilas W, Koh SS, Kim S, Srisuttee R, Cho IR, Moon J, Yoo HS, Oh S, Johnston RN and Chung YH: Cancer upregulated gene 2, a novel oncogene, enhances migration and drug resistance of colon cancer cells via STAT1 activation. Int J Oncol 43: 1111-1116, 2013.

45. Inoue-Yamauchi A, Jeng PS, Kim K, Chen HC, Han S, Ganesan YT, Ishizawa K, Jebiwott S, Dong Y, Pietanza MC, et al: Targeting the differential addiction to anti-apoptotic BCL-2 family for cancer therapy. Nat Commun 8: 16078, 2017.

46. Quinn BA, Dash R, Azab B, Sarkar S, Das SK, Kumar S, Oyesanya RA, Dasgupta S, Dent P, Grant S, et al: Targeting Mcl-1 for the therapy of cancer. Expert Opin Investig Drugs 20: 1397-1411, 2011.

47. Radhakrishnan H, Ilm K, Walther W, Shirasawa S, Sasazuki T, Daniel PT, Gillissen B and Stein U: MACC1 regulates Fas mediated apoptosis through STAT1/3 - Mcl-1 signaling in solid cancers. Cancer Lett 403: 231-245, 2017.

48. Jin X, Yu Y, Zou Q, Wang M, Cui Y, Xie J and Wang Z: MicroRNA-105 promotes epithelial-mesenchymal transition of nonsmall lung cancer cells through upregulating Mcl-1. J Cell Biochem 120: 5880-5888, 2019.

49. Zhao C, Wang Y, Jin H and Yu T: Knockdown of microRNA-203 alleviates LPS-induced injury by targeting MCL-1 in C28/I2 chondrocytes. Exp Cell Res 359: 171-178, 2017.
50. Gong J, Zhang JP, Li B, Zeng C, You K, Chen MX, Yuan Y and Zhuang SM: MicroRNA-125b promotes apoptosis by regulating the expression of Mcl-1, Bcl-w and IL-6R. Oncogene 32: 3071-3079, 2013

51. Placzek WJ, Wei J, Kitada S, Zhai D, Reed JC and Pellecchia M: A survey of the anti-apoptotic Bcl-2 subfamily expression in cancer types provides a platform to predict the efficacy of Bcl-2 antagonists in cancer therapy. Cell Death Dis 1: e40, 2010.

52. Zhang X, Zhang J, Wei H and Tian Z: STAT3-decoy oligodeoxynucleotide inhibits the growth of human lung cancer via down-regulating its target genes. Oncol Rep 17: 1377-1382, 2007.

53. Salazar-Montes A, Ruiz-Corro L, Sandoval-Rodriguez A, Lopez-Reyes A and Armendariz-Borunda J: Increased DNA binding activity of NF-kappaB, STAT-3, SMAD3 and AP-1 in acutely damaged liver. World J Gastroenterol 12: 5995-6001, 2006.

54. Townsend PA, Scarabelli TM, Davidson SM, Knight RA, Latchman DS and Stephanou A: STAT-1 interacts with p53 to enhance DNA damage-induced apoptosis. J Biol Chem 279: 5811-5820, 2004.

This work is licensed under a Creative Commons Attribution-NonCommercial-NoDerivatives 4.0 International (CC BY-NC-ND 4.0) License. 